December 20, 2013

LA-UR-13-22745

arXiv:1304.5480

\title{
A Mesonic Analog of the Deuteron
}

\author{
T. Goldman* \\ Theoretical Division, MS-B283, \\ Los Alamos National Laboratory, \\ Los Alamos, NM 87545 \\ and \\ Dept. of Physics and Astronomy, \\ University of New Mexico, \\ Albuquerque, NM 87501 \\ Richard R. Silbar ${ }^{\dagger}$ \\ Theoretical Division, MS-B283, \\ Los Alamos National Laboratory, \\ Los Alamos, NM 87545
}




\begin{abstract}
Using the LAMP model for nuclear quark structure, we calculate the binding energy and quark structure of a $B$ meson merging with a $D$ meson. The larger-than-nucleon masses of the two heavy quarks allow for a more reliable application of the Born-Oppenheimer-like approximation of the LAMP. With the absence of quark-level Pauli Exclusion Principle repulsive effects, the appearance of a bound state is unsurprising. Our variational calculation shows that the molecular, deuteron-like state structure changes rather abruptly, as the separation between the two mesons decreases, at a separation of about $0.45 \mathrm{fm}$, into a four-quark bound state, although one maintaining an internal structure rather than that of a four-quark bag. Unlike the deuteron, pion exchange does not provide any contribution to the $\approx 150 \mathrm{MeV}$ binding.
\end{abstract}

Keywords: heavy meson, four-quark, relativistic, variational, pion-less

\footnotetext{
* tgoldman@lanl.gov

$\dagger$ silbar@lanl.gov
} 


\section{INTRODUCTION}

What would nuclear physics look like without pion exchange? The long range of the nuclear force due to pion exchange between nucleons, along with the empirical short distance repulsion between nucleons, supports the established view of nuclear physics as due to the interaction of effective degrees of freedom that bear a very close resemblance to free space nucleons. Calculations of nuclear structure for small nuclei, using potential interactions fit to scattering data, succeed quite accurately.[1] Effective field theory expansions, with or without pions, claim successes [2] as well. For large nuclei, elaborations of the shell model can also reproduce experimentally known results.

However, all of these approaches ignore the internal structure of the three-quark states that are on-shell nucleons in free space but not so well defined off-shell degrees of freedom in the nucleus. In particular, the basis for off-shell nucleon form factors resembling those of on-shell nucleons is weak, and conflicts with the experimental results of deep inelastic scattering (DIS) on nuclei. Those results are not well represented by multiplying the results of DIS on free space nucleons by the number of nucleons in the target nucleus. This is known as the "EMC effect". [3]

The relativistic Los Alamos Model Potential [4, 5] (LAMP) has been used to describe the binding and structure of ${ }^{3} \mathrm{He}$ and ${ }^{4} \mathrm{He}$, including a good description [6] of the deep inelastic structure function of ${ }^{3} \mathrm{He}$. It was explicitly constructed to access the internal quark structure of the baryonic components of the nucleus without the presumption of a free space nucleon approximation. As such, except for the difficulties of carrying out calculations, it provides a less biased view (although not a systematic expansion) of the hadronic structure of nuclei than do the conventional models referred to above.

The LAMP does not describe the deuteron at all due to the very large separation of the nucleons and the dominance of single-pion exchange contributions there.[7] The LAMP, lacking quark-exchange correlations, best encompasses medium and short-range meson exchanges (two-pion, $\rho$, etc.). It must therefore be supplemented with long-range single-pion-exchange contributions [8] for a better description of nuclear binding energies.

However, in this model, we can ask: What would nuclear physics, and in particular, the deuteron, look like in the absence of long-range pion exchange interactions? If bound states exist, the constituents would be much closer together than in actual nuclei and disruption 
of the internal structure could be much more significant than suggested by the LAMP as applied to nucleons or the results of conventional nuclear physics. Could one still identify nucleonic effective degrees of freedom even when the multi-quark hadronic objects are in such close proximity that their average separation is less than their internal structure? This is to be contrasted with real nuclei where the mean separation between nucleons is quite close to twice their root-mean-square radii.

In this paper, we make an initial address to this question by considering a simpler problem, the binding of two heavy mesons. Large mass quarks are used to mimic the large mass of the nucleon, but one light antiquark in each stands in for the diquark in the nucleons and so simplifies the calculations. Since no quark-exchange correlations are included, no ( $t$-channel) quark-antiquark combinations with pion quantum numbers contribute any more significantly than higher mass mesons. However, the extension/size of the mesonic states is comparable to that of nucleons due to the spread of the light quark wavefunction.

In fact, for this case, all "light" meson exchanges are prevented, and the interactions have solely to do with the structure of the light antiquark wave functions under the influence of the color confining force, represented here by a collective potential. This is somewhat analogous, in principle, to the nuclear shell model potential although significantly different in form to be consistent with known models of confinement.

In particular, we examine here the structure of a four-quark system derived from $B^{-}=b \bar{u}$ and $D^{+}=c \bar{d}$ mesons for a bound state, or their neutral equivalents when the light antiquarks are exchanged between them. Because these mesons are considerably more massive than nucleons, localization energy is much reduced. This brings them into closer proximity than the nucleons in a deuteron, or indeed, even in a large nucleus. The larger-than-nucleon masses of the two heavy quarks also allow for a more reliable application of the BornOppenheimer-like approximation of the LAMP. Furthermore, the quark content chosen here does not involve any pairs of quarks with the same (internal) quantum numbers, so there are no (quark) Pauli exclusion effects such as those that contribute to the short-range repulsion between nucleons. Thus, this is a system in which one can expect greater accuracy of the LAMP and a significantly more deeply bound state than the deuteron.

When this $B-D$ bound state is observed, the deviation from our predictions here will provide a very good measure of the center of mass motion and breathing mode collective excitations. These are difficult to remove in the LAMP due to its relativistic nature. Since 
the non-relativistic model analogous to the LAMP, the Quark Delocalization and Color Screening Model of Wang et al. [9], gives very similar results to the LAMP after removing such effects, we expect the corrections due to these effects to be small. Thus our predictions here should be reasonably accurate.

There have been many different approaches, going back to the Cornell potential [10], along lines comparable to the LAMP, to modelling quark-antiquark states using potentials. We note here a few recent references [11]. There have also been many papers devoted to the study of four-quark systems, with a view to identifying exotic states constructed of more than three quarks or one quark and one antiquark. See, for example, the references in the recent review of Brambilla et al. [12] and some very early papers [13] as well. Generally, however, these papers have focused on states more likely to appear in hadronic collisions, such as those with the quark content of $B$ and $\bar{B}$ or $D$ and $\bar{D}$ mesons and their excited state partners (for a recent example, see [14]), since strong production of heavy quarks proceeds in a pairwise fashion. (Some, such as Ref.([13]), have also included consideration of the case studied here, albeit without the intricacies available in the LAMP). In general, the mixing of these states with the charmonium and bottomonium spectra, however, make for difficulties in extracting them unambiguously from experimental observations and may require the determination of exotic quantum numbers. No such problems occur in the case considered here, although the reduced probability of production must certainly be recognized. In any event, our interest is not in the prediction of exotic states, but in the elucidation of the origins of the nature of nuclear structure and thus a deeper understanding of it.

\section{A. Initial Concepts}

The LAMP treats the confining potential for quarks (and antiquarks) as a fixed scalar interaction in a Born-Oppenheimer-like picture, with the location of the potential minimum defining the system location. Quarks bound in a baryon or meson are treated as being bound within this potential rather than directly to each other. As such, there are immediate concerns about removing center-of-mass and breathing mode contributions to the evaluated state energy. This concern is ameliorated by comparing the energy of the interacting system of the two heavy mesons with the value at large (essentially infinite) separation.

In this paper, in addition to the confining Lorentz scalar potential of the LAMP, we 
have included a Lorentz vector potential, as is required from the observed small spin-orbit interaction in the non-relativistic quark model.[15] In fact, the vector potential is also taken as linear, attractive, but without a Coulomb-like contribution, as discussed in Ref.([16]).

In the LAMP, the confining potentials for each hadron are distributed in an array and are truncated on the mid-planes between them. While complex in general, for the case of interest here - two heavy mesons - the structure is very similar to that of the hydrogen molecule in the Born-Oppenheimer approximation, except for the linear vs. inverse distance form of the potential. In this case, the large masses of the $c$ and $b$ quarks further enhance the credibility of the approximation - they may be taken in the conventional heavy quark limit [17] as the fixed origins of the confining potentials for the light anti-quarks that complete each meson.

At large separation between the heavy quarks, confinement guarantees the isolation of the light quark wave functions from each other. However, as the two mesons approach within a distance less than a few times their root-mean-square radii, the truncation of the confining potential allows for tunneling of each light anti-quark wave function into the confinement region of the other heavy quark rather than the one to which the light anti-quark is initially bound. The concept behind this is that a quark can only be confined to nearest center of color attraction, as in string-flip models [18], for example. This spreading out, or delocalization, of the wave functions naturally reduces the localization energy and provides an initial source of binding between the two hadrons.

\section{B. Color magnetic and quantum number issues}

In nuclei and other systems, this basic consideration is complicated by additional elements: there are color 6 combinations of quarks and color-magnetic spin interactions of significance on the scale of the binding energy. Here again, the concerns raised by these considerations are considerably reduced - the color magnetic interactions between the heavy quarks are reduced by their large masses. The light quark color magnetic interactions with the heavy quarks are also reduced. Only the light-quark to light-quark color magnetic interaction remains comparable to that inferred in simple quark models of light-quark states. This energy is at most $\approx 50 \mathrm{MeV}$ as seen [19] in individual hadrons (nucleons, $\Delta$ 's, light spin-0, and spin-1 mesons) where it depends on the color and spin-strong-isospin combinations determined by the constraints of statistics. Furthermore, here the presence of both 
color 6 and color 3 combinations, as well as spin-1 and spin-0 elements, make it clear that strong cancellations of these color magnetic effects to low levels are to be expected. Therefore, in this paper we will largely ignore these contributions, since our emphasis here is to determine whether the $B$ and $D$ form a four-quark bound state or a more molecular-like combination of two identifiable mesons. We also will neglect the very small electro-magnetic contributions.

Because of these simplifications, in this paper we can also ignore the fact that there are two neutral states $\left(B^{-} D^{+}\right.$and $\left.\overline{B^{0}} D^{0}\right)$ that should exist and mix, splitting to form states of definite strong isospin ( 0 and 1$)$ although both have $I_{3}=0$. They also allow us to ignore the detailed spin structures, ranging from $J=0$ to $J=2$, the last with all of the quark spins aligned. Also unlike the individual nucleon case, the $c$ and $b$ quarks may combine antisymmetrically to form a color $\overline{\mathbf{3}}$ state or symmetrically to form a color $\mathbf{6}$. In the first case, the light anti-quarks must form a color $\mathbf{3}$ antisymmetrically, thus requiring the spin-isospin combination to be symmetric $(I=0, J=0$, or $I=1, J=1)$ and in the latter, the opposite is true - a color $\overline{\mathbf{6}}$ and $(I=1, J=0$, or $I=0, J=1)$.

Again, these allowed spin-isospin combinations for the light quarks would only produce significant energy differences if the color magnetic interaction were larger than the overall binding due to delocalization. The color $\mathbf{6}$ combination of the heavy quarks would not be expected to produce any attraction, as indeed no such components appear in baryons, but the

color $\overline{\mathbf{3}}$ combination would. Neither of these effects is included here as the channel to color neutralization by decomposition into two color-singlet mesons ( $B$ and $D$ ) is almost open, so overall color confinement issues should not be significant. In any event, symmetrization and antisymmetrization between the $c$ and $b$ quarks is moot as they are distinguishable.

We turn now to the detailed calculations of the light (anti)quark wave functions in the double well defined by the Born-Oppenheimer-fixed heavy quarks.

\section{THE TWO-WELL WAVE FUNCTION}

For two wells separated by $2 \delta$ at dimensionless positions $\mathbf{w}_{ \pm}=\{0,0, \pm \delta\}$ along the $z$-axis (see Fig. 1), we define the wave function

$$
\Psi_{L}(\mathbf{r})=\psi\left(\mathbf{r}_{-}\right)+\epsilon \psi\left(\mathbf{r}_{+}\right), \quad \text { where } \quad \mathbf{r}_{ \pm}=\mathbf{r}+\mathbf{w}_{ \pm}=\{x, y, z \pm \delta\}
$$




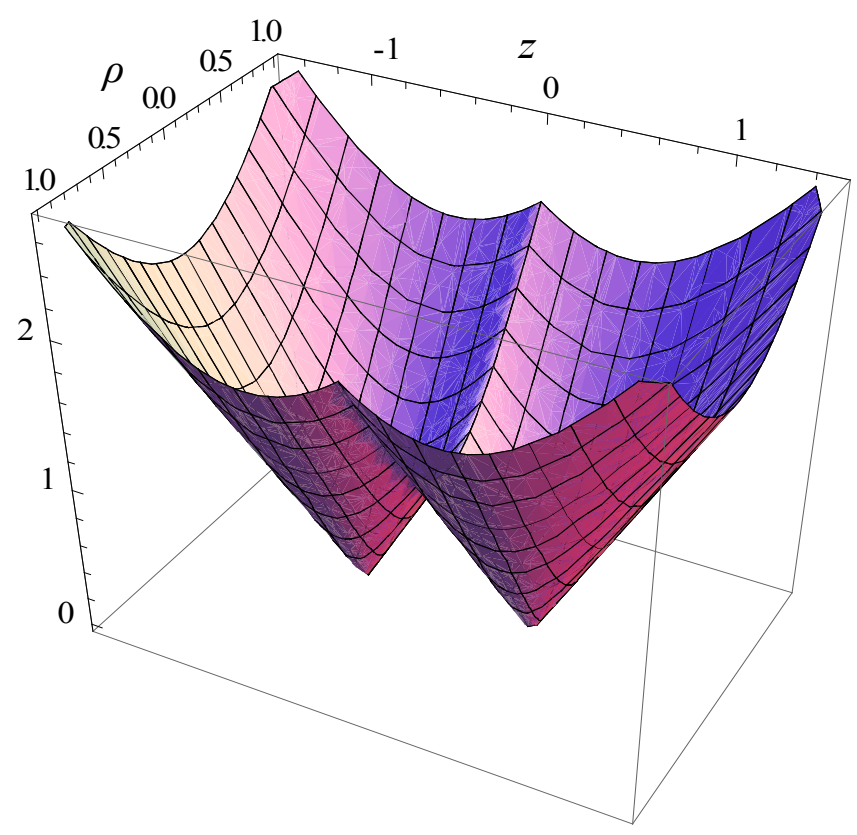

FIG. 1. Two-well linear potential. In this and all the following figures, distances, energies, and wave functions are dimensionless.

This represents, for example, a light $\bar{u}$-quark (which we assume to be massless) mostly moving and confined in the well at $r_{-}$(the "right") provided by the heavy $b$-quark. There may be some "leakage," represented by $\epsilon$, into the "left" well at $r_{+}$, provided by the heavy $c$-quark. As mentioned above, we assume that the $b$ and $c$ quark masses are large enough to justify a Born-Oppenheimer approximation of this sort. There is a similar wave function $\Psi_{R}$ with $r_{-}$and $r_{+}$interchanged in Eq. (1) for a light $\bar{d}$-quark mostly confined to the well at $r_{+}$with $\epsilon$-leakage into the well at $r_{-}$.

We will determine variationally what the best values of the parameters $\delta$ and $\epsilon$ are that provide a four-quark or molecular-like binding that form a $b \bar{u} c \bar{d}$ system. The $b$ and $c$ are well separated compared with their Compton sizes. Since they have little, if any, wave function overlap and have distinct quantum numbers, anti-symmetrization issues are irrelevant. For the rest of the paper we will drop the subscripts $L$ and $R$ on $\Psi$, but it should be borne in mind when we finally compose the $b \bar{u} c \bar{d}$ four-quark state. 
In this paper we work as much as possible with dimensionless quantities (with $\hbar=c=1$ ). That is, $\delta$, r, etc., are all dimensionless distances. The dimensionless potentials $V(r)$ and $S(r)$ given below in Eq. (4) are related to dimension-full potentials $\mathcal{V}$ and $\mathcal{S}$ by a factor of $\kappa^{2}$, which has dimensions of $\mathrm{GeV} / \mathrm{fm}$. For example, $\mathcal{S}$ would be defined as $\mathcal{S}(\mathrm{r})=\kappa^{2} \mathrm{r}$, where $r=r / \kappa$ has dimensions in fm. In GMSS [4], to cite one reference, $\kappa^{2}$ was chosen to be $0.9 \mathrm{GeV} / \mathrm{fm}$, corresponding to $\kappa=2.21 \mathrm{fm}^{-1}$. In this paper we have used a larger value, $\kappa^{2}=1.253 \mathrm{GeV} / \mathrm{fm}$, or $\kappa=2.520 \mathrm{fm}^{-1}$, as found in our fitting of charmonia masses. [16]

We take the $\psi$ 's in Eq. (11) to be dimensionless four-component Dirac wave functions for light massless $u$ - and $d$-quarks. They are solutions of

$$
H_{D} \psi=[-i \boldsymbol{\alpha} \cdot \nabla+V(\mathbf{r})+\beta S(\mathbf{r})] \psi=E \psi .
$$

Here $V(\mathbf{r})$ is the time component of a Lorentz four-vector and $S(\mathbf{r})$ is a Lorentz scalar potential (both to be specified below). With the Pauli spinor $\chi$ assumed to be quantized along the $z$-direction with spin-projection $m_{s}$, the normalized four-component $s$-wave Dirac wave function $\psi(\mathbf{r})$ is

$$
\psi_{m_{s}}(\mathbf{r})=\frac{1}{\sqrt{4 \pi}}\left(\begin{array}{c}
\psi_{a}(r) \chi_{m_{s}} \\
i \boldsymbol{\sigma} \cdot \mathbf{r} \psi_{b}(r) \chi_{m_{s}}
\end{array}\right)
$$

The upper and lower radial wave functions $\psi_{a}(r)$ and $\psi_{b}(r)$ can be chosen real. We have calculated them by solving the coupled radial Dirac equations [20] for (dimensionless) linear Lorentz vector and scalar potentials of the form

$$
V(r)=r-R \quad \text { and } \quad S(r)=r .
$$

Here $-R$ is a negative displacement pushing the vector potential $V(r)$ down below the scalar potential $S(r)$, so that confinement trumps Klein-Gordon pair creation.[15]

The curves in Fig. 2 show the calculated (dimensionless) $1 S$ wave functions $\psi_{a}(r)$ and $r \psi_{b}(r)$ when the potentials have $R=1.92, \kappa^{2}=1.253 \mathrm{GeV} / \mathrm{fm}$. Physical dimensions can be obtained by dividing the dimensionless $r$, $R$, etc., by $\kappa=2.52 \mathrm{fm}^{-1}$. The ground state eigenenergy resulting from this calculation is $0.375 \mathrm{GeV}$. These potentials provide a reasonable fit to the $c \bar{c}$ spectrum.[16] 


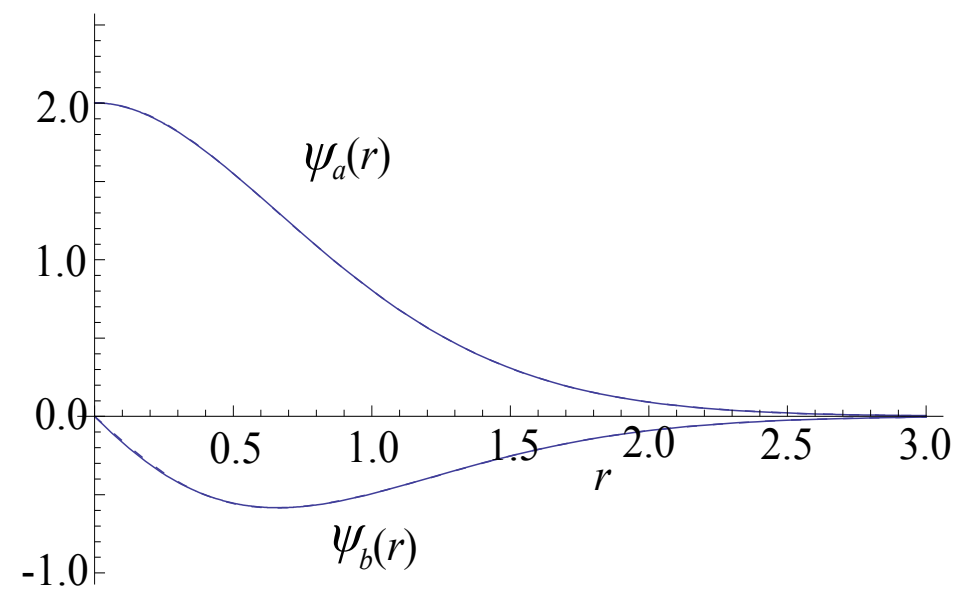

FIG. 2. Normalized massless quark $1 s$ wave functions $\psi_{a}(r)$ (above the axis) and $r \psi_{b}(r)$ (below).

\section{EXPANDING $\left\langle H_{D}{ }^{2}\right\rangle$}

The idea is that we will want to minimize the expectation value $\left\langle H_{D}^{2}\right\rangle^{1 / 2}$ with respect to the parameters $\epsilon$ and $\delta$ to bound (approximately) the energy for the four-quark system consisting of $b, c, \bar{u}$, and $\bar{d}$. The square $\left\langle H_{D}^{2}\right\rangle$ is required for a variational bound as, due to negative energy states, $\left\langle H_{D}\right\rangle$ itself is unbounded below. The Dirac Hamiltonian $H_{D}$ is displayed in Eq. (2) but now, for the two-well case (Fig. 1), the potentials are

$$
V(\mathbf{r})=\left\{\begin{array}{ll}
r_{-}-R, & \text { if } z>0 \\
r_{+}-R, & \text { if } z<0
\end{array} \quad \text { and } \quad S(\mathbf{r})=\left\{\begin{array}{ll}
r_{-}, & \text {if } z>0 \\
r_{+}, & \text {if } z<0
\end{array} .\right.\right.
$$

As already mentioned, $-R$ is a negative offset so the vector potential lies below the scalar.

The exact two-well energy $E$ is in principle found by solving for the eigenvalue of

$$
H_{D} \Psi(\mathbf{r})=E \Psi(\mathbf{r})
$$

with $\Psi$ given in Eq. (1). This being difficult, we instead chose to find an approximate value of the four-quark energy $E$ by the above-mentioned minimization of $\left\langle H_{D}^{2}\right\rangle^{1 / 2}$.

After some algebra one finds

$$
\begin{aligned}
H_{D}^{2}=- & \nabla^{2}+V^{2}(\mathbf{r})+S^{2}(\mathbf{r})+2 \beta V(\mathbf{r}) S(\mathbf{r}) \\
& -i \boldsymbol{\alpha} \cdot[(\nabla V(\mathbf{r}))+\beta(\nabla S(\mathbf{r}))]-2 i V(\mathbf{r}) \boldsymbol{\alpha} \cdot \nabla .
\end{aligned}
$$

The lack of a term like $-2 i S(\mathbf{r}) \boldsymbol{\alpha} \cdot \nabla$ is because of a cancellation (the Dirac operators $\boldsymbol{\alpha}$ and $\beta$ anti-commute). The first four terms of $H_{D}^{2}$ are "diagonal" (generically, $\mathcal{O}_{D}$ ) in that they 


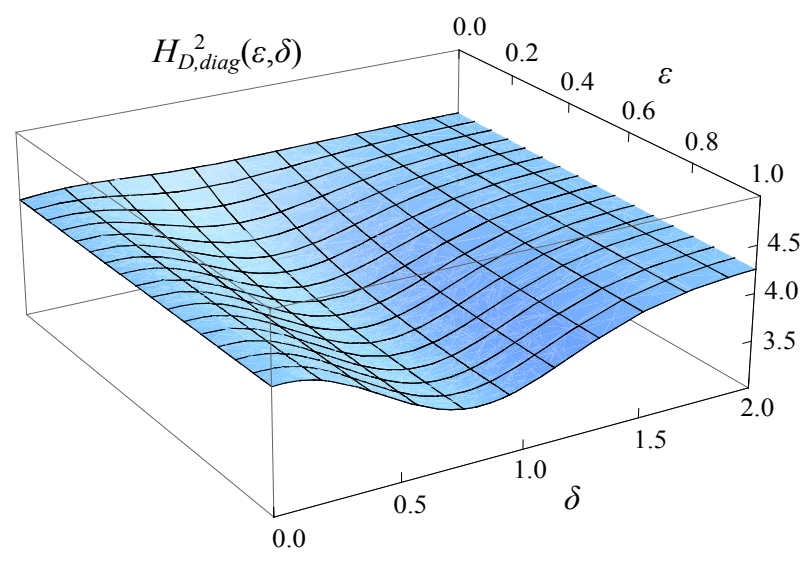

FIG. 3. Plot of all the diagonal contributions to $\left\langle H_{D}{ }^{2}(\epsilon, \delta)>\right.$.

connect $\psi_{a}$ to $\psi_{a}$ and $\psi_{b}$ to $\psi_{b}$, while the last two terms are "off-diagonal" $\left(\mathcal{O}_{O D}\right)$ connecting $\psi_{a}$ to $\psi_{b}$.

An Appendix describes, in detail, how we calculate the expectation values of the terms in Eq. (7). For brevity, we now present the numerical results of these calculations for $H_{D}{ }^{2}$ and its components.

\section{PLOTTING $<H_{D}{ }^{2}>$ TO FIND A MINIMUM ENERGY}

We combine all the expectation integrals discussed in the Appendix together to get an analytic expression for $\left\langle H_{D}{ }^{2}>\right.$, which we can plot to look for a minimum squared energy.

First, we define the (unnormalized) contribution, as a function of $\epsilon$ and $\delta$, from the diagonal pieces,

$$
\begin{array}{r}
<H_{D, \operatorname{diag}}^{2}(\epsilon, \delta)>=\sum_{i, j} a_{i} a_{j}\left[\left(1+\epsilon^{2}\right)\left(I_{<\nabla^{2}>}^{(0)}+4 I_{i j,<r_{ \pm}^{2}>}^{(0)}-4 R I_{i j,<r_{ \pm}>}^{(0)}+R^{2} I_{i j,<1>}^{(0)}\right)\right. \\
\left.+\epsilon\left(I_{i j,<\nabla^{2}>}^{(1)}+4 I_{i j,<r_{ \pm}^{2}>}^{(1)}-4 R I_{i j,<r_{ \pm}>}^{(1)}+R^{2} I_{i j,<1>}^{(1)}\right)\right] \\
+\sum_{i, j} b_{i} b_{j}\left[\left(1+\epsilon^{2}\right)\left(J_{i j,<\nabla^{2}>}^{(0)}+R^{2} J_{i j,<1>}^{(0)}\right)+\epsilon\left(J_{i j,<\nabla^{2}>}^{(1)}+R^{2} J_{i j,<1>}^{(1)}\right)\right],
\end{array}
$$

using the expressions for the integrals $I$ and $J$ given in the Appendix.

Figure 3 displays a three-dimensional plot of the normalized $<H_{D \text {, diag }}{ }^{2}(\epsilon, \delta)>/ N^{2}(\epsilon, \delta)$, where $N^{2}(\epsilon, \delta)$ is also discussed and displayed in the Appendix. It shows a relatively shallow minimum at $\epsilon=1$ and $\delta \approx 0.8$. Note the large value, a dimensionless squared-energy of $\approx 4$, 


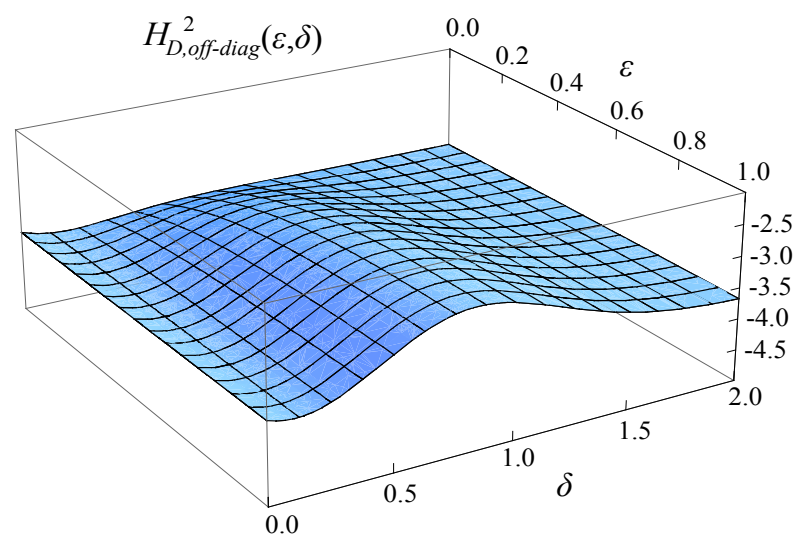

FIG. 4. Plot of all the off-diagonal contributions to $\left\langle H_{D}{ }^{2}(\epsilon, \delta)>\right.$.

which must be largely cancelled by the off-diagonal contributions to achieve a squared-energy similar to that for the one-well case, $E^{2}=0.5685$.

The off-diagonal (unnormalized) contributions are

$$
\begin{array}{r}
<H_{D, \text { off-diag }}^{2}(\epsilon, \delta)>=\sum_{i, j} a_{i} b_{j}\left[\left(1+\epsilon^{2}\right)\left(K_{i j,<\nabla V S>}^{(0)}+K_{i j,<V \nabla>}^{(0)}\right)\right. \\
\left.+\epsilon\left(K_{i j,<\nabla V S>}^{(1)}+K_{i j,<V \nabla>}^{(1)}\right)\right],
\end{array}
$$

with integrals $K$ also from the Appendix. Figure 4 gives the three-dimensional plot of $<H_{D, \text { off-diag }}^{2}(\epsilon, \delta)>/ N^{2}(\epsilon, \delta)$. In contrast with $<H_{D, \text { diag }}^{2}>/ N^{2}$, it has a repulsive hump around $\delta \approx 1$ as well as a shallow valley running from $\epsilon=0$ to 1 for $\delta \approx 0.2$. In the final sum of diagonal and off-diagonal contributions that hump will fill in the minimum seen in Fig. 3.

Thus we finally combine the two contributions, defining a normalized

$$
<H_{D}^{2}(\epsilon, \delta)>=\left[<H_{D, \text { off-diag }}^{2}(\epsilon, \delta)>+<H_{D, \text { off-diag }}^{2}(\epsilon, \delta)>\right] / N^{2}(\epsilon, \delta)
$$

Figure 5 plots how $H_{D}{ }^{2}$, as a function of $\epsilon$ and $\delta$, develops a long, flat valley for all values of $\epsilon$ at a separation of $\delta \approx 0.2$ (i.e., recalling the value of $\kappa$, a separation of $\approx 0.45 \mathrm{fm}$ ). Also important is the hump (reminiscent of a fission barrier) around $\delta \approx 0.9$ that will help to confine this four-quark system at $\delta \approx 0.2$. This hump corresponds to a repulsion between two $Q-\bar{q}$ asymptotic meson states preventing the light quarks from delocalizing. There is very little, if any, barrier to coalescence at $\epsilon=0$. 


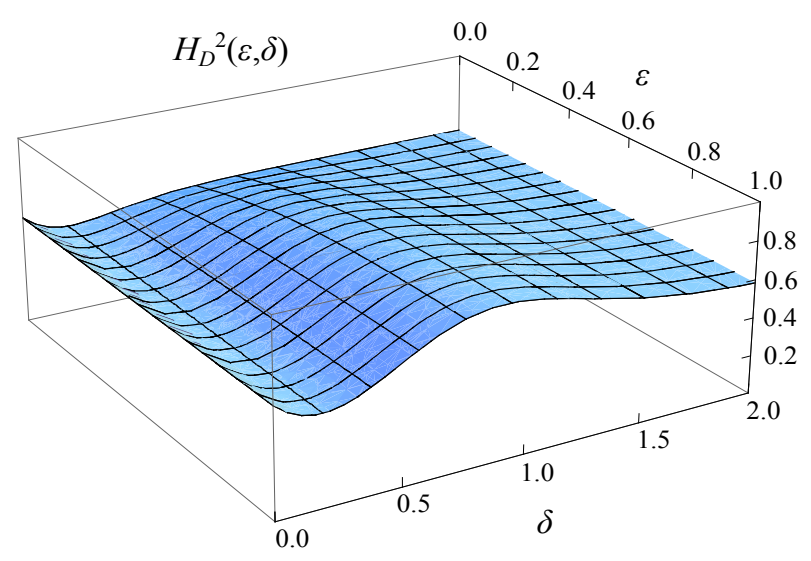

FIG. 5. Plot of the final $<H_{D}^{2}(\epsilon, \delta)>$.

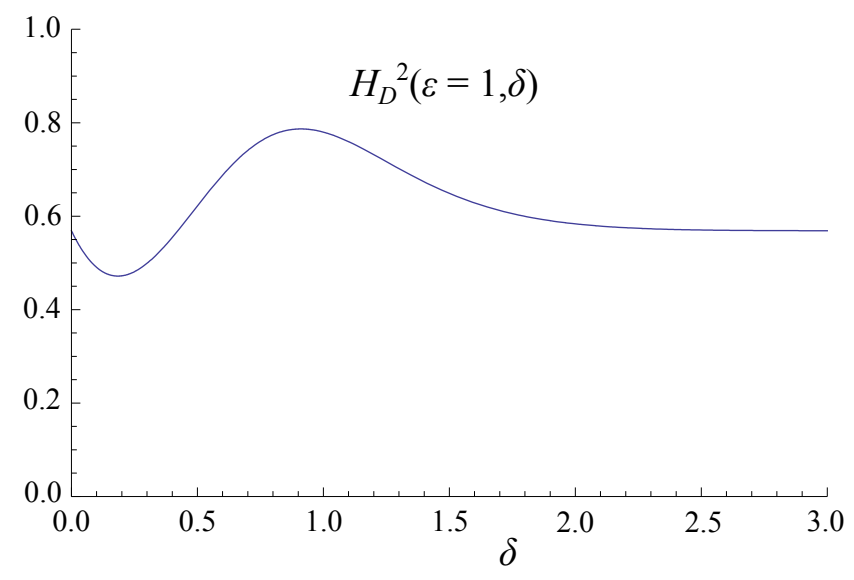

FIG. 6. $H_{D}{ }^{2}(\epsilon=1, \delta)$, with a valley at $\delta=0.18$ and a "fission barrier" at $\delta \approx 0.9$.

It is easier to see this behavior with a two-dimensional plot, Fig. 6, showing $H_{D}{ }^{2}$ as a function of $\delta$ at $\epsilon=1$, where the valley is deepest and the hump is highest.

The dimensionless squared-energy valley-depth at $\epsilon=1.0$ and $\delta=0.18, \Delta H_{D}{ }^{2}=0.097$, corresponds to a binding energy of $155 \mathrm{MeV}$ for this $b c \bar{u} \bar{d}$ four-quark mesonic state. The valley is surprisingly flat, as shown in Fig. 7, dropping only 0.0023 squared dimensionless energy units from $\epsilon=0$ to $\epsilon=1$. This corresponds to an energy drop of about $24 \mathrm{MeV}$, a rather small energy difference. This suggests that Zitterbewegung may play an important role in the nature of this meson. 


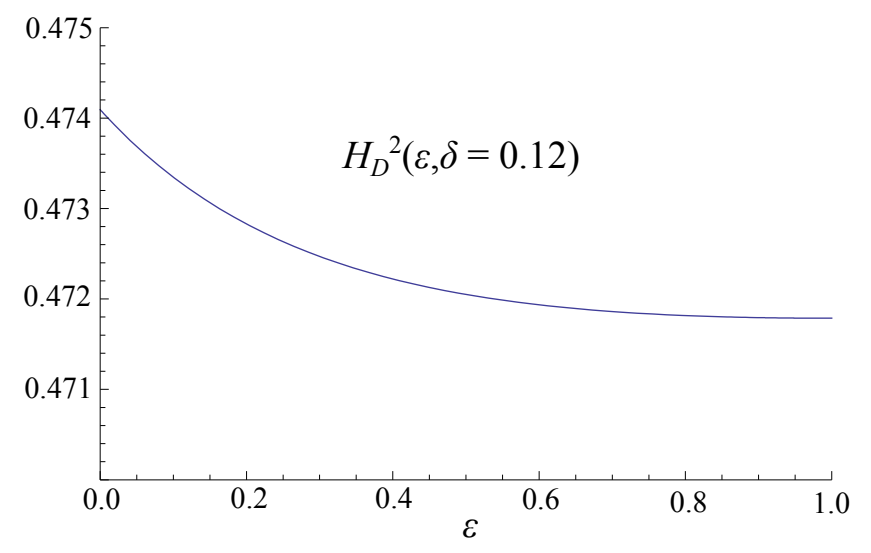

FIG. 7. Plot of how the nearly flat valley at $\delta=0.18$ decreases from $\epsilon=0$ to $\epsilon=1$.

\section{DISCUSSION}

Figure 8 is a contour plot of the binding energy of the state in the $\epsilon-\delta$ plane. It displays two remarkable features: The first is that, at very small $\epsilon$, appropriate to the approach towards each other of the two asymptotic $(B$ and $D)$ mesons, there is no evidence of a repulsive barrier to the fusion of those mesons. The second is that the valley of attraction at small meson separation is very flat between small $\epsilon(\sim 0.2)$ and $\epsilon=1$. This indicates that there is little energy associated with fluctuations in the $\epsilon$ collective variable of the light quarks in the state. There may be a more significant amount associated with the $\delta$ collective variable, but this effect is suppressed by the large masses associated with the Born-Oppenheimer centers defined by the heavy quarks, at least when viewed non-relativistically as seems appropriate for them, due to their relatively large masses. We therefore expect little correction to our estimates of the mass of the four-quark state due to collective variable effects.

The dashed curve in Figure 8 illustrates how two well-separated $B$ and $D$ mesons at $\epsilon=0$ and large $\delta$ would come together to $\delta \approx 0.2$ and $\epsilon \approx 0.2$, corresponding to a heavy quark separation of about $0.45 \mathrm{fm}$. As we have emphasized above, this small separation makes it clear that long-range pion-exchange effects do not contribute significantly. From $\epsilon \approx 0.2$, the four-quark state then slides gently down the nearly flat valley to $\epsilon=1$ where it is most bound. Such a state is prevented from falling apart because of the "fission barrier" around $\delta \approx 0.9$. 


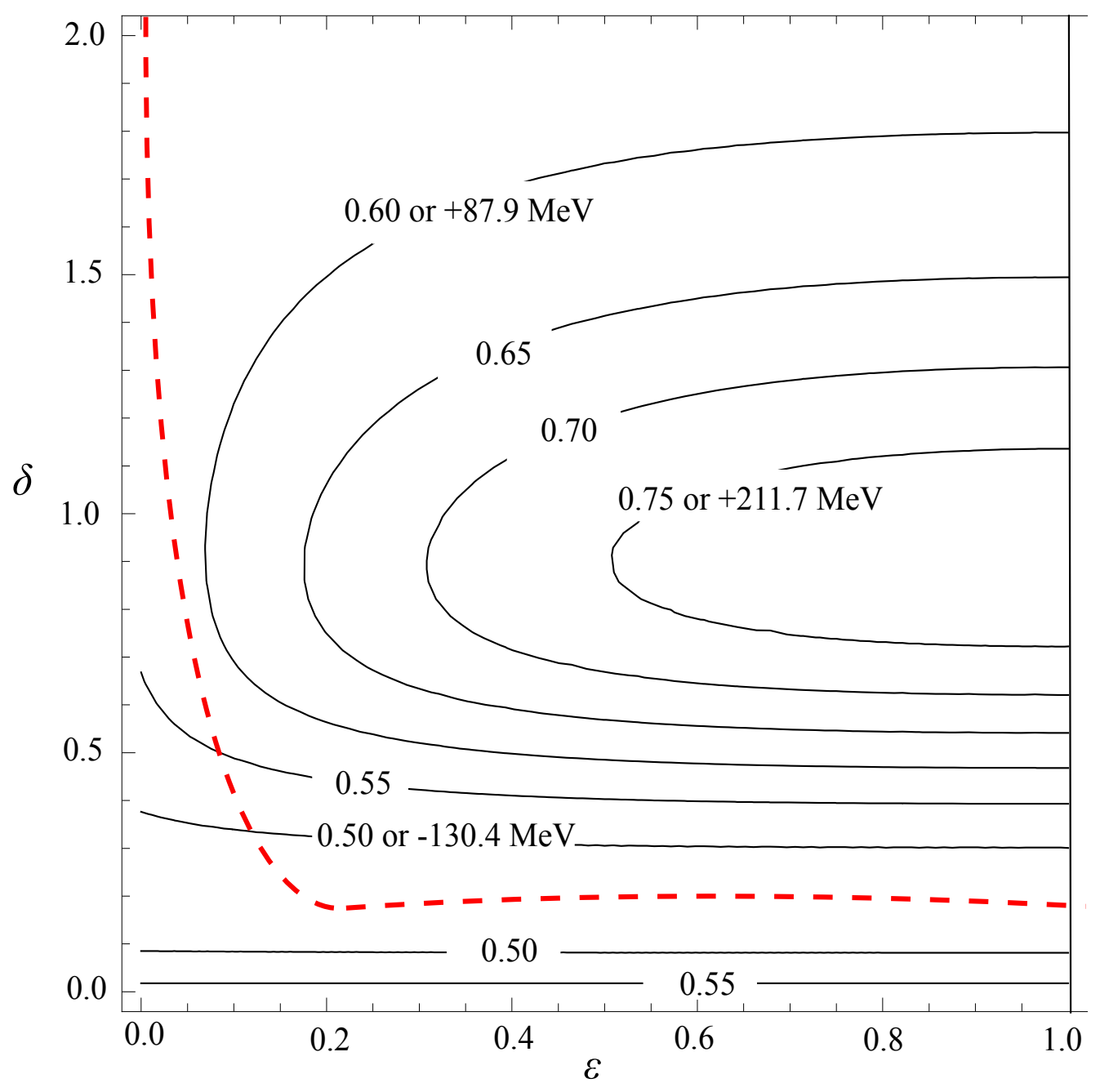

FIG. 8. Contour plot of $H_{D}{ }^{2}(\epsilon, \delta)$. The dashed curve illustrates how two well-separated $Q-\bar{q}$ mesons at $\epsilon=0$ and large $\delta$ come together and slide down the valley at $\delta \approx 0.2$ to form a four-quark state at $\epsilon=1$.

We have ignored the possible color magnetic contributions from the interaction of the two light antiquarks, but this must be less than $50 \mathrm{MeV}$ and we expect it to be even less than half this value. These corrections, which we will deal with in a future publication, are not large compared to the extracted variational upper bound on the binding energy of order 150 $\mathrm{MeV}$ found in our calculations. Thus, by comparing our binding energy with the threshold for $B$ and $D$ mesons, we predict a set of states in the region of $7 \mathrm{GeV} / c^{2}$.

Finally, we comment on the surprisingly small difference in binding energy between the 
"molecular" form of the bound state, $(\epsilon \approx 0.2$, as in nuclei [4])) and the four-quark limit $(\epsilon=1)$. If this feature is widespread in such heavy quark systems, it could go far towards explaining why it has been so difficult to identify unambiguous four-quark states.

In any event, as our interest here is in nuclear physics, we note that the small separation compared to root-mean-square size of the meson states argues against the identification of the system as that of two slightly off-shell free space mesons, at least, at $\epsilon \sim 1$. However, the small difference in energy between that region and $\epsilon \sim 0.2$ suggests to the contrary, that since the binding energy is not large, at least some of the time, the system would appear to be one described as two slightly off-shell free space mesons, with substantial fluctuations between the two pictures. Difficult as it was historically, we conclude that nuclear physics would have been even more difficult to understand if it had similar properties.

\section{ACKNOWLEDGMENTS}

This work was carried out in part under the auspices of the National Nuclear Security Administration of the U.S. Department of Energy at Los Alamos National Laboratory under Contract No. DE-AC52-06NA25396.

[1] Steven C. Pieper, R. B. Wiringa, and J. Carlson, Phys. Rev. C 70 (2004) 054325-1.

[2] See, e.g., U. van Kolck, Prog. Part. Nucl. Phys. 43 (1999) 337.

[3] European Muon Collaboration (J. Ashman et al.), Z.Phys.C57 (1993) 211 and earlier papers cited there.

[4] T. Goldman, K. R. Maltman, G. J. Stephenson, Jr., and K. E. Schmidt, Nucl. Phys. A481 (1988) 621.

[5] C. J. Benesh, T. Goldman, and G. J. Stephenson, Jr., Phys. Rev. C48 (1993) 1379 and Phys. Rev. C68 (2003) 045208.

[6] C. J. Benesh and T. Goldman, Phys. Rev. C55, (1997) 441.

[7] See for example, J. L. Friar, B. F. Gibson and G. L. Payne, Phys. Rev. C30 (1984) 1084.

[8] E.g., T. Goldman and R. R. Silbar, Phys. Rev. C77 (2008) 065203.

[9] Fan Wang, Guang-han Wu, Li-jian Teng, and T. Goldman, Phys. Rev. Lett. 69 (1992) 2901. 
[10] E. Eichten, K. Gottfried, T. Kinoshita, K. D. Lane and T. -M. Yan, Phys. Rev. D17 (1978) 3090.

[11] Some recent examples include: Takayuki Matsuki and Koichi Seo, Phys. Rev. D85 (2012) 014036; Stanislaw D. Glazek, "Hypothesis of quark binding by condensation of gluons in hadrons", presented at LIGHTCONE 2011, 23 - 27 May, 2011, Dallas, TX, arXiv:1110.1430 Jin-Hee Yoon, Byeong-Noh Kim, Horace W. Crater and Cheuk-Yin Wong, "On the Mass Difference between pion and rho meson using a Relativistic Two-Body Model", Talk presented at the Fifth Asia-Pacific Conference on Few-Body Problems in Physics, August 22-26, 2011, Seoul, Republic of Korea, to be published in Few-Body Systems, arXiv:1110.1598; M. Blank and A. Krassnigg, Phys. Rev. D84 (2011) 096014.

[12] N. Brambilla, et al., Eur. Phys. J. C71 (2011) 1534.

[13] L. Heller and J. A. Tjon, Phys. Rev. D35 (1987) 969; J. A. Carlson, L. Heller and J. A. Tjon, Phys. Rev. D37 (1988) 744.

[14] Carlos Peña and David Blaschke, Acta Phys. Pol. B, Proc. Suppl. 5 (2012 963).

[15] P. R. Page, T. Goldman, and J. N. Ginocchio, Phys. Rev. Lett. 86 (2001) 204.

[16] T. Goldman and R. R. Silbar, Phys. Rev. C85 (2012) 015203.

[17] N. Isgur and M. B. Wise, Phys. Lett. B237 (1990) 527, Phys. Rev. D43 (1991) 819, Phys. Rev. Lett. 66 (1991) 1130, and Nucl. Phys. B48 (1991) 276.

[18] See, e.g., M. Oka, Phys. Rev. D31 (1985) 2274, Phys. Rev. D31 (1985) 2773, and subsequent related articles.

[19] J. Beringer et al. (Particle Data Group), Phys. Rev. D86 (2012) 010001. A convenient access to this data is to go on-line to http://pdglive.lbl.gov/.

[20] R. R. Silbar and T. Goldman, Eur. J. Phys. 32 (2011) 217. 


\section{Appendix A: Calculational Details}

\section{Approximating $\psi_{a}$ and $\psi_{b}$ as a sum of Gaussians}

For the calculations presented below, the $\psi_{a, b}$ have both been fitted to sums of Gaussians,

$$
\psi_{a}(r)=\sum_{i} a_{i} e^{-\mu_{i} r^{2} / 2}, \quad \psi_{b}(r)=\sum_{i} b_{i} e^{-\mu_{i} r^{2} / 2},
$$

where the $a_{i}, b_{i}$, and $\mu_{i}$ are dimensionless numbers. We found it necessary to go to six terms, so that evaluating the upper and lower components of the left-hand-side of the Dirac equation [Eq. (2) in the main text] gives reasonable agreement with the right-hand-side. The fitted parameters are

$$
\begin{aligned}
& \left.\mu_{i}=1.0,1.3,1.6,2.0,4.0,8.0\right\} \\
& a_{i}=\{0.492649,-0.687482,1.84609,-0.00246039,0.258295,0.0956581\} \\
& b_{i}=\{-0.0571296,1.03367,-1.18398,1.33989,0.162575,0.299479\} .
\end{aligned}
$$

The fitted $\psi_{a}(r)$ and $r \psi_{b}(r)$ are shown as the dashed curves in Fig. 2, largely overlying the solid curves from the solution of the Dirac equation. To check the quality of the fits we have evaluated the single-quark expectation value of the Hamiltonian, $\left\langle H_{D}\right\rangle=0.7545$, which is slightly larger than the (dimensionless) energy eigenvalue $E=0.7540$ (which, for a variational trial function, is as it should be). As a second check on our Gaussian fits of $\psi_{a}$ and $\psi_{b}$, Eqs. (A1) and (4), we also evaluated the single-well expectation $\left\langle H_{D}^{2}>\right.$ to be 0.5691, again slightly larger than $E^{2}=0.5685$, as it should be.

\section{General Remarks on calculating the expectations}

The reason for approximating our numerical radial wave functions $\psi_{a}$ and $\psi_{b}$ as sums of Gaussians is that it allows us to calculate the expectation values of each of the terms of $H_{D}^{2}$ analytically. Given an analytic expression for $H_{D}^{2}$ allows us to plot it quickly and precisely as a function of the variational parameters $\delta$ and $\epsilon$. To do these integrations, we have relied heavily on programs such as Mathematica and Maple. As will be seen, the final results can sometimes be messy and often involve error functions ${ }^{1}$ because of the Gaussians being integrated.

${ }^{1}$ See, e.g., M. Abramovitz and I. A. Stegun, Handbook of Mathematical Functions, (Dover, New York, 1965), Chap. 7 
For the diagonal operators of $H_{D}^{2}$ we will calculate the upper and lower contributions separately,

$$
\left\langle\Psi\left|\mathcal{O}_{D}\right| \Psi\right\rangle=\left\langle\Psi\left|\mathcal{O}_{D}\right| \Psi\right\rangle_{A}+\left\langle\Psi\left|\mathcal{O}_{D}\right| \Psi\right\rangle_{B}
$$

The $B$-expectations are more complicated than those for $A$ because of the factors of $-i \boldsymbol{\sigma} \cdot \mathbf{r}_{ \pm}$ multiplying the radial $\psi_{b}$ 's. However, for some diagonal operators, as will be seen below, the $B$-expectations are not always needed. In any case, from Eq. A1 we expand these diagonal operator expectations as

$$
\left\langle\Psi\left|\mathcal{O}_{D}\right| \Psi\right\rangle_{A}=\sum_{i, j} a_{i} a_{j} I_{i j}, \quad\left\langle\Psi\left|\mathcal{O}_{D}\right| \Psi\right\rangle_{B}=\sum_{i, j} b_{i} b_{j} J_{i j}
$$

where the $I_{i j}$ and $J_{i j}$ are integrals over Gaussians.

First, we separate out the quadratic dependence on $\epsilon$ as

$$
I_{i j}=I_{i j}^{(0)}+\epsilon I_{i j}^{(1)}+\epsilon^{2} I_{i j}^{(2)}=\left(1+\epsilon^{2}\right) I_{i j}^{(0)}+\epsilon I_{i j}^{(1)}
$$

and likewise for the lower-component $B$-integrals $J_{i j}$. The second equality here comes about because parity symmetry ensures that the $I_{i j}^{(2)}=I_{i j}^{(0)}$, etc. We will refer to the $I_{i j}^{(0)}$ as "direct terms," in that they connect Gaussians with $\mu_{j} r_{-}^{2} / 2$ to those with $\mu_{i} r_{-}^{2} / 2$ (and similarly for $I_{i j}^{(2)}$ with $\left.r_{+}\right)$. Recalling the $1 / 4 \pi$ from the normalization of the $\psi$ 's, we ensure the symmetry under the interchange of indices $i$ and $j$ by writing

$$
I_{i j}^{(0)}=\frac{1}{8 \pi} \int d^{3} r\left\{e^{-\mu_{i} r_{-}^{2} / 2} \mathcal{O}_{D} e^{-\mu_{j} r_{-}^{2} / 2}+e^{-\mu_{j} r_{-}^{2} / 2} \mathcal{O}_{D} e^{-\mu_{i} r_{-}^{2} / 2}\right\} .
$$

The direct integrals $J_{i j}^{(0)}$ have a similar form but with $\boldsymbol{\sigma} \cdot \mathbf{r}_{-} \mathcal{O}_{D} \boldsymbol{\sigma} \cdot \mathbf{r}_{-}$in place of the $\mathcal{O}_{D}$.

The "cross terms" $I_{i j}^{(1)}$ are more complicated integrals than the $I_{i j}^{(0)}$, and likewise for $J_{i j}^{(1)}$. They connect Gaussians with $\mu_{j} r_{-}^{2} / 2$ to $\mu_{j} r_{+}^{2} / 2$ and vice versa. Thus, on symmetrizing in $i$ and $j$,

$$
\begin{aligned}
& I_{i j}^{(1)}=\frac{1}{8 \pi} \int d^{3} r\left\{\left[e^{-\mu_{i} r_{-}^{2} / 2} \mathcal{O}_{D} e^{-\mu_{j} r_{+}^{2} / 2}+e^{-\mu_{i} r_{+}^{2} / 2} \mathcal{O}_{D} e^{-\mu_{j} r_{-}^{2} / 2}+\right]\right. \\
&\left.+\left[e^{-\mu_{j} r_{-}^{2} / 2} \mathcal{O}_{D} e^{-\mu_{i} r_{+}^{2} / 2}+e^{-\mu_{j} r_{+}^{2} / 2} \mathcal{O}_{D} e^{-\mu_{i} r_{-}^{2} / 2}\right]\right\}
\end{aligned}
$$

The $J_{i j}^{(1)}$ have a similar form but with $\mathcal{O}_{D}$ replaced by $\boldsymbol{\sigma} \cdot \mathbf{r}_{-} \mathcal{O}_{D} \boldsymbol{\sigma} \cdot \mathbf{r}_{+}$or $\boldsymbol{\sigma} \cdot \mathbf{r}_{+} \mathcal{O}_{D} \boldsymbol{\sigma} \cdot \mathbf{r}_{-}$, as appropriate.

Each of the off-diagonal operators in Eq. (9) of the main text has the general form

$$
\mathcal{O}_{O D}=-i \boldsymbol{\alpha} \cdot \mathbf{X}=\left[\begin{array}{cc}
0 & -i \boldsymbol{\sigma} \cdot \mathbf{X}_{12} \\
-i \boldsymbol{\sigma} \cdot \mathbf{X}_{21} & 0
\end{array}\right]
$$


where the $\mathbf{X}_{12}$ and $\mathbf{X}_{21}$ are vector-operators that may not be equal because of the possible presence of the diagonal $\beta$ matrix in $\mathcal{O}_{O D}$.

The direct terms of the off-diagonal expectation $\left\langle\mathcal{O}_{O D}>\right.$ involve several terms because the upper component of $\Psi^{\dagger}\left(\mathbf{r}_{-}\right)$connects through $-i \boldsymbol{\sigma} \cdot \mathbf{X}_{12}$ to the lower component of $\Psi\left(\mathbf{r}_{-}\right)$at the same time that the lower component of $\Psi^{\dagger}\left(\mathbf{r}_{-}\right)$connects through $-i \boldsymbol{\sigma} \cdot \mathbf{X}_{21}$ to the upper component of $\Psi\left(\mathbf{r}_{-}\right)$. We therefore have to keep the sums over the $a$ 's and $b$ 's in Eq. (A1) as parts of the integrand. Again symmetrizing in $i$ and $j$,

$$
\begin{aligned}
<\mathcal{O}_{O D}^{(0)}>=\frac{1}{8 \pi} \sum_{i, j} \int d^{3} r\left\{e^{-\mu_{i} r_{-}^{2} / 2}\right. & {\left[-a_{i} b_{j}\left(\boldsymbol{\sigma} \cdot \mathbf{X}_{12}\right)\left(\boldsymbol{\sigma} \cdot \mathbf{r}_{-}\right)\right.} \\
& \left.+a_{j} b_{i}\left(\boldsymbol{\sigma} \cdot \mathbf{r}_{-}\right)\left(\boldsymbol{\sigma} \cdot \mathbf{X}_{21}\right)\right] e^{-\mu_{j} r_{-}^{2} / 2} \\
+e^{-\mu_{j} r_{-}^{2} / 2} & {\left[-a_{j} b_{i}\left(\boldsymbol{\sigma} \cdot \mathbf{X}_{12}\right)\left(\boldsymbol{\sigma} \cdot \mathbf{r}_{-}\right)\right.} \\
& \left.\left.+a_{i} b_{j}\left(\boldsymbol{\sigma} \cdot \mathbf{r}_{-}\right)\left(\boldsymbol{\sigma} \cdot \mathbf{X}_{21}\right)\right] e^{-\mu_{i} r_{-}^{2} / 2}\right\} .
\end{aligned}
$$

The cross terms of $\left\langle\mathcal{O}_{O D}>\right.$ have even more terms because the $\Psi^{\dagger}\left(\mathbf{r}_{+}\right)$connects to $\Psi\left(\mathbf{r}_{-}\right)$ at the same time that $\Psi^{\dagger}\left(\mathbf{r}_{-}\right)$connects to $\Psi\left(\mathbf{r}_{+}\right)$. It becomes

$$
\begin{aligned}
<\mathcal{O}_{O D}^{(1)}>=\frac{1}{8 \pi} \sum_{i, j} \int d^{3} r\left\{e^{-\mu_{i} r_{+}^{2} / 2}\right. & {\left[-a_{i} b_{j}\left(\boldsymbol{\sigma} \cdot \mathbf{X}_{12}\right)\left(\boldsymbol{\sigma} \cdot \mathbf{r}_{-}\right)\right.} \\
& \left.+a_{j} b_{i}\left(\boldsymbol{\sigma} \cdot \mathbf{r}_{+}\right)\left(\boldsymbol{\sigma} \cdot \mathbf{X}_{21}\right)\right] e^{-\mu_{j} r_{-}^{2} / 2} \\
+e^{-\mu_{i} r_{-}^{2} / 2} & {\left[-a_{i} b_{j}\left(\boldsymbol{\sigma} \cdot \mathbf{X}_{12}\right)\left(\boldsymbol{\sigma} \cdot \mathbf{r}_{+}\right)\right.} \\
& \left.+a_{j} b_{i}\left(\boldsymbol{\sigma} \cdot \mathbf{r}_{-}\right)\left(\boldsymbol{\sigma} \cdot \mathbf{X}_{21}\right)\right] e^{-\mu_{j} r_{+}^{2} / 2} \\
+e^{-\mu_{j} r_{+}^{2} / 2} & {\left[-a_{j} b_{i}\left(\boldsymbol{\sigma} \cdot \mathbf{X}_{12}\right)\left(\boldsymbol{\sigma} \cdot \mathbf{r}_{-}\right)\right.} \\
& \left.+a_{i} b_{j}\left(\boldsymbol{\sigma} \cdot \mathbf{r}_{+}\right)\left(\boldsymbol{\sigma} \cdot \mathbf{X}_{21}\right)\right] e^{-\mu_{i} r_{-}^{2} / 2} \\
+e^{-\mu_{j} r_{-}^{2} / 2} & {\left[-a_{j} b_{i}\left(\boldsymbol{\sigma} \cdot \mathbf{X}_{12}\right)\left(\boldsymbol{\sigma} \cdot \mathbf{r}_{+}\right)\right.} \\
& \left.\left.+a_{i} b_{j}\left(\boldsymbol{\sigma} \cdot \mathbf{r}_{-}\right)\left(\boldsymbol{\sigma} \cdot \mathbf{X}_{21}\right)\right] e^{-\mu_{j} r_{+}^{2} / 2}\right\}
\end{aligned}
$$

The integrations for the $I$ 's, $J$ 's, and in Eqs. (A9) and (A10) can best be done using (dimensionless) cylindrical coordinates, $\rho=\left(x^{2}+y^{2}\right)^{1 / 2}, \theta$, and $z$. The $\theta$ integrations are trivial, providing a factor of $2 \pi$, which will cancel with the $1 / 4 \pi$ coming from the normalizations of the $\psi$ 's in Eq. (3) to give an overall factor of $1 / 2$ before each double integral over $\rho$ and $z$. It usually is easier to do the $\rho$-integration (from 0 to $+\infty$ ) first. Because $V(\mathbf{r}$ ) and $S(\mathbf{r})$ depend on $r_{-}$when $z>0$ and on $r_{+}$when $z<0$, we need to do the $z$-integration separately for those regions, i.e., for $z$ from $-\infty$ to 0 and then for $z$ from 0 to $+\infty$. The separate results are then added and simplified to give the final integral. 
We will distinguish the results for the expectations of the different operators in Eq. (7) by an appropriate subscript. For example, for $O_{D}=\nabla^{2}$, we will write $I_{i j}^{(0,1)}$ as $I_{i j,<\nabla^{2}>}^{(0,1)}$, and similarly for the $J_{i j}$ integrals.

\section{Normalizing $\Psi$}

While the Dirac $\psi$ 's are themselves properly normalized, the two-well $\Psi$ is not. For this we need to calculate the expectation values of $\mathcal{O}_{D}=1$ to find

$$
N^{2}(\delta, \epsilon)=\int d^{3} r \Psi^{\dagger} \Psi=\langle\Psi|1| \Psi\rangle=\langle\Psi|1| \Psi\rangle_{A}+\langle\Psi|1| \Psi\rangle_{B}
$$

We make the expansion in $\epsilon$ as in Eq. (A5) above. The direct-term integrals for the expectation $\langle 1\rangle$ are, noting that for the $J_{i j,<1>}^{(0)}$ we also have a factor of $\left(\boldsymbol{\sigma} \cdot \mathbf{r}_{-}\right)\left(\boldsymbol{\sigma} \cdot \mathbf{r}_{-}\right)=r_{-}^{2}$ in the integrand,

$$
\begin{aligned}
I_{i j,<1>}^{(0)} & =\left[\frac{\pi}{2\left(\mu_{i}+\mu_{j}\right)^{3}}\right]^{1 / 2} \\
J_{i j,<1>}^{(0)} & =3\left[\frac{\pi}{2\left(\mu_{i}+\mu_{j}\right)^{5}}\right]^{1 / 2},
\end{aligned}
$$

both independent of $\delta$.

The cross-term integrals do depend on $\delta$. For the $J_{i j,<1>}^{(1)}$ we need the factor

$$
\left(\boldsymbol{\sigma} \cdot \mathbf{r}_{+}\right)\left(\boldsymbol{\sigma} \cdot \mathbf{r}_{-}\right)=\mathbf{r}_{+} \cdot \mathbf{r}_{-}=\rho^{2}+z^{2}-\delta^{2}
$$

in the integrand. Proceeding as in Sec. A 2, we find

$$
\begin{aligned}
I_{i j,<1>}^{(1)} & =\left[\frac{2 \pi}{\left(\mu_{i}+\mu_{j}\right)^{3}}\right]^{1 / 2} e^{-2 \mu_{i} \mu_{j} \delta^{2} /\left(\mu_{i}+\mu_{j}\right)}, \\
J_{i j,<1>}^{(1)} & =\left[3\left(\mu_{i}+\mu_{j}\right)-4 \mu_{i} \mu_{j} \delta^{2}\right]\left[\frac{2 \pi}{\left(\mu_{i}+\mu_{j}\right)^{7}}\right]^{1 / 2} e^{-2 \mu_{i} \mu_{j} \delta^{2} /\left(\mu_{i}+\mu_{j}\right)} .
\end{aligned}
$$

Note that, when $\delta=0, I_{i j,<1>}^{(1)}=2 I_{i j,<1>}^{(0)}$, and $J_{i j,<1>}^{(1)}=2 J_{i j,<1>}^{(0)}$. This is a common feature for all the expectations here and below. This is necessary so that, for example, when $\delta=0$ and $\epsilon=1$, one recovers a result that is four times that when $\delta=0$ and $\epsilon=0$.

We see from Eq. A15 that $I_{i j,<1>}^{(1)}$, as a function of $\delta$, is a decaying Gaussian (as in Fig. 9 . plot A). On the other hand, $J_{i j,<1>}^{(1)}$ falls off from its peak at $\delta=0$, goes through zero, and has a mild minimum before decaying to zero at large $\delta^{2}$ (as in Fig. 9, plot B). 

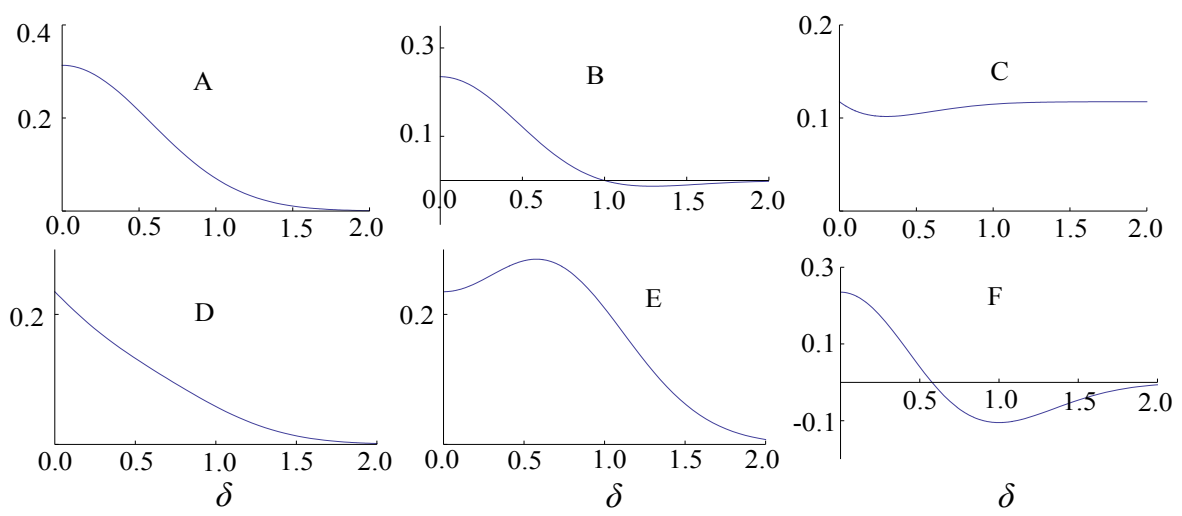

FIG. 9. Typical plots of $I$ 's, $J$ 's, and $K$ 's as functions of $\delta$. The $y$-axes are in arbitrary units.

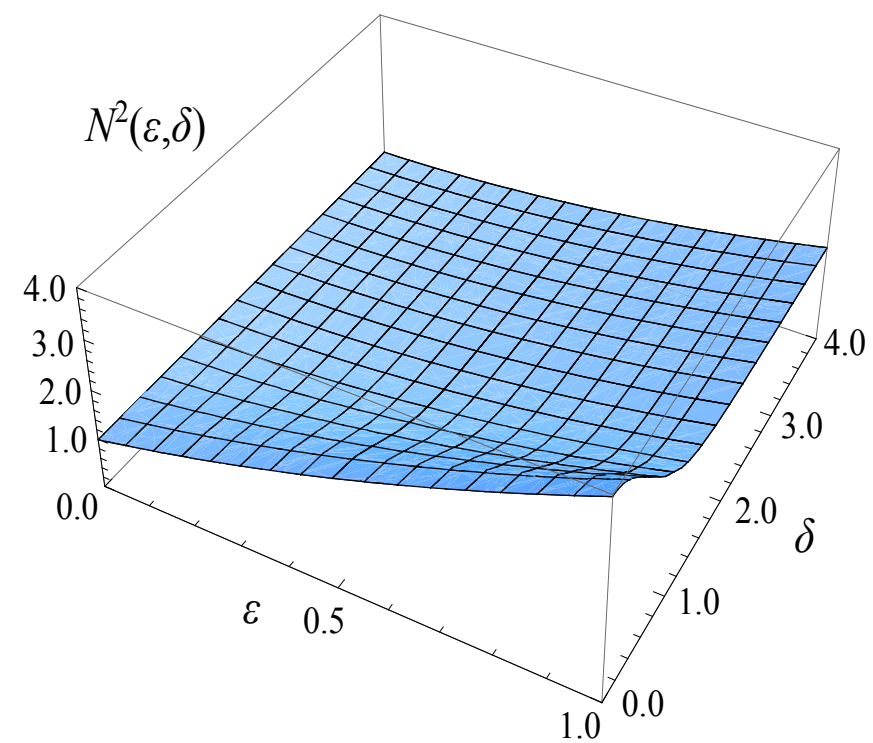

FIG. 10. Three-dimensional plot of $N^{2}(\epsilon, \delta)$.

Combining all terms,

$$
N^{2}(\epsilon, \delta)=\sum_{i, j} a_{i} a_{j}\left[\left(1+\epsilon^{2}\right) I_{<1>}^{(0)}+\epsilon I_{<1>}^{(1)}\right]+\sum_{i, j} b_{i} b_{j}\left[\left(1+\epsilon^{2}\right) J_{<1>}^{(0)}+\epsilon J_{<1>}^{(1)}\right]
$$

and the normalized $\Psi$ is obtained by multiplying Eq. (1) by $1 / N(\epsilon, \delta)$.

Figure 10 shows a plot of $N^{2}(\epsilon, \delta)$ for the values of the $a$ 's, $b$ 's, and $\mu$ 's that were fitted to the normalized $\psi_{a}$ and $\psi_{b}$, Eq. A2). We have checked that, for these values, $N^{2}(0,0)=$ $0.9858 \approx 1$ and $N^{2}(1,0)=3.9430 \approx 4$, as they should but with some deviation $(\approx 2 \%)$ coming from the inexactness of the fitting. The ratio of the two values is 4 to high accuracy. 


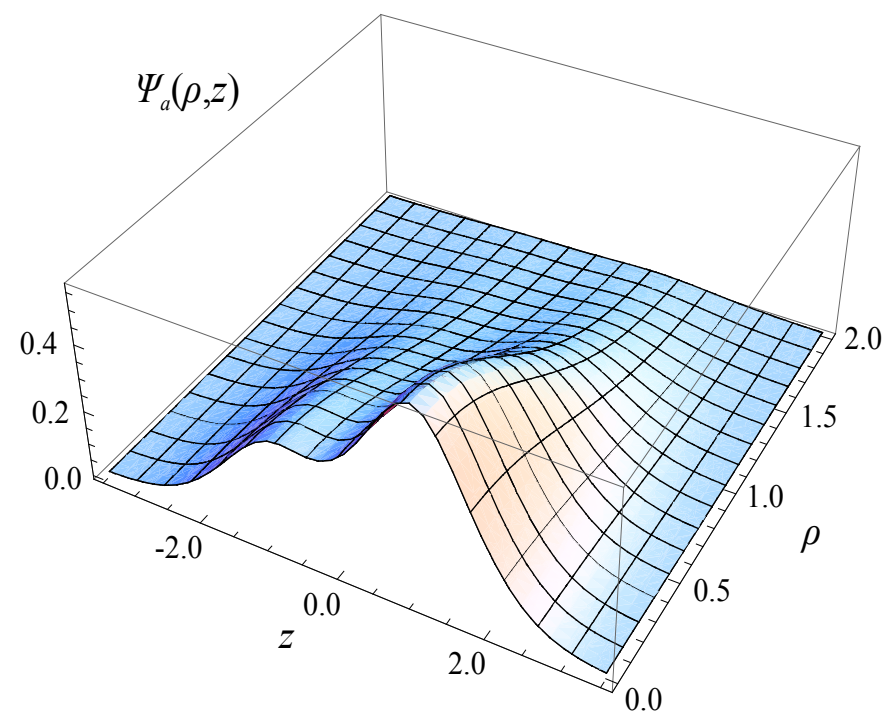

FIG. 11. Plot of a normalized $\Psi_{a}(\rho, z)$ for $\epsilon=0.5$ and $\delta=1.0$.

To illustrate what "leakage" from one well to the other might look like, Fig. 11 shows a plot of the upper component of the normalized $\Psi$ as a function of $\rho$ (running from 0 to 2) and $z$ (running from -3.5 to +3.5 ) for $\epsilon=0.5$ and $\delta=1.1$.

\section{Evaluating the diagonal expectation $\left\langle-\nabla^{2}\right\rangle$}

First, note that, for $\mathbf{r}_{ \pm}=\{x, y, z \pm \delta\}$, the $i$ th component of the gradient

$$
\nabla_{i}=\frac{\partial}{\partial x_{i}}=\nabla_{i}^{\prime}=\frac{\partial}{\partial x_{i}^{\prime}} \quad \text { for } \quad \mathbf{r}^{\prime}=\left\{x^{\prime}=x, y^{\prime}=y, z^{\prime}=z \pm \delta\right\}=\mathbf{r}_{ \pm}
$$

since each $\partial x_{i}^{\prime} / \partial x_{i}=1$. Thus we can replace the result of the Laplacian with respect to $r$ acting on a function such as $\psi_{a}\left(r_{-}\right)$with that for a Laplacian with respect to $r_{-}$acting on that function. For spherical coordinates, $-\nabla^{2}$ on the angle-independent $e^{-\mu_{j} r_{-}^{2} / 2}$ then becomes

$$
-\nabla^{2} e^{-\mu_{j} r_{-}^{2} / 2}=-\nabla^{\prime 2} e^{-\mu_{j} r_{-}^{2} / 2}=-\frac{1}{r_{-}} \frac{d^{2}}{d r_{-}^{2}}\left(r_{-} e^{-\mu_{j} r_{-}^{2} / 2}\right)=-\mu_{j}\left(\mu_{j} r_{-}^{2}-3\right) e^{-\mu_{j} r_{-}^{2} / 2},
$$

whence the three-dimensional integral reduces, after symmetrizing and cancelling factors of $4 \pi$, to

$$
I_{i j,<-\nabla^{2}>}^{(0)}=3 \mu_{i} \mu_{j}\left[\frac{\pi}{2\left(\mu_{i}+\mu_{j}\right)^{5}}\right]^{1 / 2}
$$


independent of $\delta$.

For the $B$-integrals things are more complicated because of the $\boldsymbol{\sigma} \cdot \mathbf{r}_{-}$factor to the right of the Laplacian. After some algebra,

$$
-\left(\boldsymbol{\sigma} \cdot \mathbf{r}_{-} \nabla^{2} \boldsymbol{\sigma} \cdot \mathbf{r}_{-}\right) e^{-\mu_{j} r_{-}^{2} / 2}=-r_{-}^{2} \mu_{j}\left(\mu_{j} r_{-}^{2}-5\right) e^{-\mu_{j} r_{-}^{2} / 2}
$$

whence

$$
J_{i j,<-\nabla^{2}>}^{(0)}=15 \mu_{i} \mu_{j}\left[\frac{\pi}{2\left(\mu_{i}+\mu_{j}\right)^{7}}\right]^{1 / 2}
$$

also independent of $\delta$.

The cross terms, again, do depend on $\delta$.

$$
I_{i j,<-\nabla^{2}>}^{(1)}=\mu_{i} \mu_{j}\left[3\left(\mu_{i}+\mu_{j}\right)-4 \mu_{i} \mu_{j} \delta^{2}\right]\left[\frac{2 \pi}{\left(\mu_{i}+\mu_{j}\right)^{7}}\right]^{1 / 2} e^{-2 \mu_{i} \mu_{j} \delta^{2} /\left(\mu_{i}+\mu_{j}\right)} .
$$

This integral as a function of $\delta$ looks like Fig. $9 \mathrm{~B}$.

For the corresponding $B$-cross term, one proceeds in the same manner but, instead of Eq. A21, we need ${ }^{2}$

$$
-\left(\boldsymbol{\sigma} \cdot \mathbf{r}_{+} \nabla^{2} \boldsymbol{\sigma} \cdot \mathbf{r}_{-}\right) e^{-\mu_{j} r_{-}^{2} / 2}=-\left(\rho^{2}+z^{2}-\delta^{2}\right) \mu_{j}\left(\mu_{j} r_{-}^{2}-5\right) e^{-\mu_{j} r_{-}^{2} / 2} .
$$

We find

$$
\begin{gathered}
J_{i j,<-\nabla^{2}>}^{(1)}=\mu_{i} \mu_{j}\left[15\left(\mu_{i}+\mu_{j}\right)^{2}-40 \mu_{i} \mu_{j}\left(\mu_{i}+\mu_{j}\right) \delta^{2}+16 \mu_{i}^{2} \mu_{j}^{2} \delta^{4}\right] \times \\
{\left[\frac{2 \pi}{\left(\mu_{i}+\mu_{j}\right)^{11}}\right]^{1 / 2} e^{-2 \mu_{i} \mu_{j} \delta^{2} /\left(\mu_{i}+\mu_{j}\right)} .}
\end{gathered}
$$

This integral as a function of $\delta$ also looks like Fig. $9 \mathrm{~B}$, but because it is quartic, it is slightly positive beyond $\delta=1.7$.

\section{Evaluating the expectation of $V^{2}+S^{2}+2 \beta V S$}

This is also a diagonal operator. The linear vector potential $V(\mathbf{r})$ differs from the linear scalar potential $S(\mathbf{r})$ by a negative offset $-R$. In $\left\langle V^{2}(\mathbf{r})\right\rangle$ the integrals of $\left\langle r_{ \pm}^{2}\right\rangle$ are the same as those for $\left\langle S^{2}(\mathbf{r})\right\rangle$. Here, $\left\langle r_{ \pm}^{2}\right\rangle$ means the integration of $r_{-}^{2}$ when $z>0$ and of $r_{+}^{2}$ when $z<0$. Thus we (schematically) expand the diagonal $V^{2}+S^{2}+2 \beta V S$ as

$$
\left\langle V^{2}+S^{2}+2 \beta V S\right\rangle=2\left\langle r_{ \pm}^{2}\right\rangle(1+\beta)-2 R\left\langle r_{ \pm}\right\rangle(1+\beta)+R^{2}\langle 1\rangle
$$

2 Because we have separated the two wells along the $z$-direction, the cross product $\mathbf{r}_{+} \times \mathbf{r}_{-}$only has $x$ and $y$ components. Since we have assumed the Pauli spinor $\chi_{m_{s}}$ to be polarized along the $z$-axis, the term from the product of two Pauli $\sigma$ matrices that gives a $i \boldsymbol{\sigma} \cdot \mathbf{r}_{+} \times \mathbf{r}_{-}$contribution vanishes. 
The factor of $(1+\beta)$ ensures that only the upper components of $\Psi$ contribute to the first two expectation values. That is, we only need to calculate the $A$-integrals (the $I$ 's) for those terms. The expectation value $\langle 1\rangle$ multiplying $R^{2}$ does have contributions from the lower components and their integrals $I_{<1>}^{(0)}, I_{<1>}^{(1)}, J_{<1>}^{(0)}$, and $J_{<1>}^{(1)}$ are given in Sec. A 3. The integrals for the operators $\left\langle r_{ \pm}^{2}\right\rangle$ and $\left\langle r_{ \pm}\right\rangle$are rather more complicated and their analytic forms are presented next.

a. Expectation of $\mathcal{O}_{D}=r_{ \pm}^{2}$

The direct integral for this operator is

$$
\begin{aligned}
I_{i j,<r_{ \pm}^{2}>}^{(0)}= & -\frac{2 \delta}{\left(\mu_{i}+\mu_{j}\right)^{2}} e^{-\left(\mu_{i}+\mu_{j}\right) \delta^{2} / 2} \\
& +\left[\frac{\pi}{2\left(\mu_{i}+\mu_{j}\right)^{5}}\right]^{1 / 2}\left[3+2\left(\mu_{i}+\mu_{j}\right) \delta^{2} \operatorname{Erfc}\left(\sqrt{\frac{\left(\mu_{i}+\mu_{j}\right)}{2}} \delta\right)\right] .
\end{aligned}
$$

Note the linear dependence on $\delta$, which gives rise to a shallow minimum near the origin before the function returns to its initial value, as in Fig. $9 \mathrm{C}$.

The cross-term integral for $\left\langle r_{ \pm}^{2}\right\rangle$ is

$$
\begin{aligned}
I_{i j,<r_{ \pm}^{2}>}^{(1)}= & -\frac{4 \delta}{\left(\mu_{i}+\mu_{j}\right)^{2}} e^{-\left(\mu_{i}+\mu_{j}\right) \delta^{2} / 2} \\
+ & {\left[\frac{2 \pi}{\left(\mu_{i}+\mu_{j}\right)^{7}}\right]^{1 / 2} e^{-2 \mu_{i} \mu_{j} \delta^{2} /\left(\mu_{i}+\mu_{j}\right)} \times } \\
& \left\{3\left(\mu_{i}+\mu_{j}\right)+2\left(\mu_{i}^{2}+\mu_{j}^{2}\right) \delta^{2}-2\left(\mu_{i}^{2}-\mu_{j}^{2}\right) \delta^{2} \operatorname{Erf}\left(\frac{\left(\mu_{i}-\mu_{j}\right)}{\sqrt{2\left(\mu_{i}+\mu_{j}\right)}} \delta\right)\right\}
\end{aligned}
$$

which also has odd terms in $\delta$. In this case, as a function of $\delta, I_{i j,<r_{ \pm}^{2}>}^{(1)}$ falls off smoothly to zero from its peak value at $\delta=0$, as in Fig. 9 D. $I_{i j,<r_{ \pm}^{2}>}^{(1)}$ is symmetric in $i$ and $j$ because $\operatorname{Erf}(-x)=-\operatorname{Erf}(x), I_{i j,<r_{ \pm}^{2}>}^{(1)}=I_{j i,<r_{ \pm}^{2}>}^{(1)}$. Also, as expected,

$$
I_{<r_{ \pm}^{2}>}^{(1)}=2 I_{<r_{ \pm}^{2}>}^{(0)}=3\left[\frac{2 \pi}{\left(\mu_{i}+\mu_{j}\right)^{5}}\right]^{1 / 2}
$$

when $\delta=0$. 
b. Expectation of $\mathcal{O}_{D}=r_{ \pm}$

The direct term for this operator is

$$
\begin{aligned}
I_{i j,<r_{ \pm}>}^{(0)}= & \frac{1}{2\left(\mu_{i}+\mu_{j}\right)^{2}}\left[4+2 e^{-2\left(\mu_{i}+\mu_{j}\right) \delta^{2}}-3 e^{-\left(\mu_{i}+\mu_{j}\right) \delta^{2} / 2}\right] \\
-\frac{1}{2 \delta}\left[\frac{\pi}{2\left(\mu_{i}+\mu_{j}\right)^{5}}\right]^{1 / 2} \times & \\
& \left\{\left(\mu_{i}+\mu_{j}\right) \delta^{2}-\left(1+4\left(\mu_{i}+\mu_{j}\right) \delta^{2}\right) \operatorname{Erf}\left(\sqrt{2\left(\mu_{i}+\mu_{j}\right)} \delta\right)\right. \\
& \left.+\left(1+3\left(\mu_{i}+\mu_{j}\right) \delta^{2}\right) \operatorname{Erf}\left(\sqrt{\left(\mu_{i}+\mu_{j}\right) / 2} \delta\right)\right\} .
\end{aligned}
$$

This integral also has an odd term in $\delta$, like $I_{i j,<r_{ \pm}^{2}>^{-}}^{(0)}$ As a function of $\delta$ it resembles that shown in Fig. 9 C. That is, despite the $1 / \delta$ factor in the last term, $I_{i j,<r_{ \pm}>}^{(0)}$ is not singular at $\delta=0$ (i.e., when there is no separation between the two wells): $I_{i j,<r_{ \pm}>}^{(0)} \rightarrow 2 /\left(\mu_{i}+\mu_{j}\right)^{2}$ as $\delta \rightarrow 0$.

The cross term for $\left\langle r_{ \pm}\right\rangle$is

$$
\begin{aligned}
I_{i j,<r_{ \pm}>}^{(1)}= & \frac{2}{\left(\mu_{i}+\mu_{j}\right)^{2}}\left(e^{-2 \mu_{i} \delta^{2}}+e^{-2 \mu_{j} \delta^{2}}-e^{-\frac{1}{2}\left(\mu_{i}+\mu_{j}\right) \delta^{2}}\right) \\
+ & \frac{1}{2 \delta \mu_{i} \mu_{j}}\left[\frac{\pi}{2\left(\mu_{i}+\mu_{j}\right)^{5}}\right]^{1 / 2}\left\{\left(\mu_{i}+\mu_{j}\right)^{2} \operatorname{Erfc}\left(\sqrt{\frac{\mu_{i}+\mu_{j}}{2}} \delta\right)\right. \\
& -2 \mu_{j}\left(\mu_{i}+\mu_{j}+4 \mu_{i}^{2} \delta^{2}\right) e^{-\frac{2 \delta^{2} \mu_{i} \mu_{j}}{\mu_{i}+\mu_{j}}} \operatorname{Erfc}\left(\sqrt{\frac{2}{\mu_{i}+\mu_{j}}} \mu_{i} \delta\right) \\
& +2 \mu_{i}\left(\mu_{i}+\mu_{j}+4 \mu_{j}^{2} \delta^{2}\right) e^{-\frac{2 \delta^{2} \mu_{i} \mu_{j}}{\mu_{i}+\mu_{j}}} \operatorname{Erf}\left(\sqrt{\frac{2}{\mu_{i}+\mu_{j}}} \mu_{j} \delta\right) \\
& \left.-\left(\mu_{i}-\mu_{j}\right)\left(\mu_{i}+\mu_{j}-4 \mu_{i} \mu_{j} \delta^{2}\right) e^{-\frac{2 \delta^{2} \mu_{i} \mu_{j}}{\mu_{i}+\mu_{j}}} \operatorname{Erfc}\left(\frac{\left(\mu_{i}-\mu_{j}\right) \delta}{\sqrt{2\left(\mu_{i}+\mu_{j}\right)}}\right)\right\}
\end{aligned}
$$

Note that $I_{i j,<r_{ \pm}>}^{(1)}$ is also symmetric under the interchange of $i$ and $j$ and, again, at $\delta=0$, we have $I_{i j,<r_{ \pm}>}^{(1)}=4 /\left(\mu_{i}+\mu_{j}\right)^{2}=2 I_{i j,<r_{ \pm}>}^{(0)}$. Its behavior as a function of $\delta$ is similar to that shown in Fig. 9D, again partly due to the presence of odd terms in $\delta$.

\section{The off-diagonal expectation of $-i \boldsymbol{\alpha} \cdot[(\nabla V(\mathbf{r}))+\beta(\nabla S(\mathbf{r}))]$}

For the linear potentials of Eq. (5)

$$
\nabla V(\mathbf{r})=\nabla S(\mathbf{r})= \begin{cases}\hat{\mathbf{r}}_{-} & \text {if } z>0 \\ \hat{\mathbf{r}}_{+} & \text {if } z<0\end{cases}
$$


and we again have a simplification from the $(1+\beta)$, namely,

$$
-i \boldsymbol{\alpha} \cdot[(\nabla V(\mathbf{r}))+\beta(\nabla S(\mathbf{r}))]=-i \boldsymbol{\alpha} \cdot \hat{\mathbf{r}}_{ \pm}(1+\beta)=\left[\begin{array}{cc}
0 & 0 \\
-2 i \boldsymbol{\sigma} \cdot \hat{\mathbf{r}}_{ \pm} & 0
\end{array}\right]
$$

i.e., the operator $\mathbf{X}_{12}$ in Eq. (A8) vanishes and $\mathbf{X}_{21}$ is doubled. The latter operator connects the upper component of $\Psi^{\dagger}$ to the lower component of $\Psi$.

For the direct terms, Eq. A9 reduces to two terms

$$
\begin{aligned}
<[\nabla V S]^{(0)}>= & -2 \frac{1}{4 \pi} \sum_{i, j} \int d^{3} r\left\{e^{-\mu_{i} r_{-}^{2} / 2}\left[a_{j} b_{i}\left(\boldsymbol{\sigma} \cdot \mathbf{r}_{-}\right)\left(\boldsymbol{\sigma} \cdot \hat{\mathbf{r}}_{ \pm}\right)\right] e^{-\mu_{j} r_{-}^{2} / 2}\right. \\
& \left.+e^{-\mu_{j} r_{-}^{2} / 2}\left[a_{i} b_{j}\left(\boldsymbol{\sigma} \cdot \mathbf{r}_{-}\right)\left(\boldsymbol{\sigma} \cdot \hat{\mathbf{r}}_{ \pm}\right)\right] e^{-\mu_{i} r_{-}^{2} / 2}\right\} \\
= & \sum_{i, j}\left[a_{j} b_{i} K_{i j,<\nabla V S>}^{(0)}+a_{i} b_{j} K_{j i,<\nabla V S>}^{(0)}\right]
\end{aligned}
$$

where

$$
K_{i j,<\nabla V S>}^{(0)}=-2 \frac{1}{4 \pi} \int d^{3} r e^{-\mu_{i} r_{-}^{2} / 2}\left[\left(\boldsymbol{\sigma} \cdot \mathbf{r}_{-}\right)\left(\boldsymbol{\sigma} \cdot \hat{\mathbf{r}}_{ \pm}\right)\right] e^{-\mu_{j} r_{-}^{2} / 2}
$$

The Pauli matrices here reduce to

$$
\left(\boldsymbol{\sigma} \cdot \mathbf{r}_{-}\right)\left(\boldsymbol{\sigma} \cdot \hat{\mathbf{r}}_{ \pm}\right)=r_{-}\left(\hat{\mathbf{r}}_{-} \cdot \hat{\mathbf{r}}_{ \pm}\right)
$$

For the integration over $z>0$ the integrand becomes simply $r_{-}$, which is the same as that already needed for getting to the final result for $I_{<r_{ \pm}>}^{(0)}$ in subsection A $5 \mathrm{~b}$ above. For the integration over negative $z$, however, Eq. A36 becomes

$$
r_{-}\left(\hat{\mathbf{r}}_{-} \cdot \hat{\mathbf{r}}_{+}\right)=\mathbf{r}_{-} \cdot \mathbf{r}_{+} / r_{+}=\left(\rho^{2}+z^{2}-\delta^{2}\right) / \sqrt{\rho^{2}+(z+\delta)^{2}}
$$

which involves a new integrand, but which nonetheless can still be done analytically. (Here it is much easier to do the $\rho$-integration first.) We find

$$
\begin{aligned}
K_{i j,<\nabla V S>}^{(0)}= & -\frac{2}{\left(\mu_{i}+\mu_{j}\right)^{2}}\left[2-e^{-\left(\mu_{i}+\mu_{j}\right) \delta^{2} / 2}\right] \\
& -\frac{1}{\delta}\left[\frac{2 \pi}{\left(\mu_{i}+\mu_{j}\right)^{5}}\right]^{1 / 2}\left[\operatorname{Erf}\left(\sqrt{2\left(\mu_{i}+\mu_{j}\right)} \delta\right)-\operatorname{Erf}\left(\sqrt{\left(\mu_{i}+\mu_{j}\right) / 2} \delta\right)\right]
\end{aligned}
$$

This result is, again, symmetric and non-singular with $K_{i j,<\nabla V S>}^{(0)}=-4 /\left(\mu_{i}+\mu_{j}\right)^{2}$ at $\delta=0$. In this case there are no odd terms (!) in $\delta$. Versus $\delta$ it is similar to that shown in Fig. $9 \mathrm{C}$, but with the initial slope at the origin being zero. 
Because $K_{i j,<\nabla V S>}^{(0)}=K_{j i,<\nabla V S>}^{(0)}$, we can finally write the direct term contributions for this expectation as

$$
<[\nabla V S]^{(0)}>=\sum_{i, j}\left(a_{j} b_{i}+a_{i} b_{j}\right) \quad K_{i j,<\nabla V S>}^{(0)}
$$

regaining explicit symmetry.

The cross term integral $K_{<\nabla V S\rangle}^{(1)}$ is more complicated but is done similarly. As $\mathbf{X}_{12}=0$, there are now four terms remaining from Eq. A10,

$$
\begin{aligned}
& <[\nabla V S]^{(1)}>=-2 \frac{1}{4 \pi} \sum_{i, j} \int d^{3} r\left\{e^{-\mu_{i} r_{+}^{2} / 2}\left[a_{j} b_{i}\left(\boldsymbol{\sigma} \cdot \mathbf{r}_{+}\right)\left(\boldsymbol{\sigma} \cdot \hat{\mathbf{r}}_{ \pm}\right)\right] e^{-\mu_{j} r_{-}^{2} / 2}\right. \\
& +e^{-\mu_{i} r_{-}^{2} / 2}\left[a_{j} b_{i}\left(\boldsymbol{\sigma} \cdot \mathbf{r}_{-}\right)\left(\boldsymbol{\sigma} \cdot \hat{\mathbf{r}}_{ \pm}\right)\right] e^{-\mu_{j} r_{+}^{2} / 2} \\
& +e^{-\mu_{j} r_{+}^{2} / 2}\left[a_{i} b_{j}\left(\boldsymbol{\sigma} \cdot \mathbf{r}_{+}\right)\left(\boldsymbol{\sigma} \cdot \hat{\mathbf{r}}_{ \pm}\right)\right] e^{-\mu_{i} r_{-}^{2} / 2} \\
& \left.+e^{-\mu_{j} r_{-}^{2} / 2}\left[a_{i} b_{j}\left(\boldsymbol{\sigma} \cdot \mathbf{r}_{-}\right)\left(\boldsymbol{\sigma} \cdot \hat{\mathbf{r}}_{ \pm}\right)\right] e^{-\mu_{i} r_{+}^{2} / 2}\right\} \\
& =\sum_{i, j} \int d^{3} r\left[a_{j} b_{i} K_{i j,<\nabla V S>}^{(1)}+a_{i} b_{j} K_{j i,<\nabla V S>}^{(1)}\right] \text {, }
\end{aligned}
$$

where

$$
\begin{aligned}
& K_{i j,<\nabla V S>}^{(1)}=-2 \frac{1}{4 \pi} \int d^{3} r\left\{e^{-\mu_{i} r_{+}^{2} / 2}\left[\left(\boldsymbol{\sigma} \cdot \mathbf{r}_{+}\right)\left(\boldsymbol{\sigma} \cdot \hat{\mathbf{r}}_{ \pm}\right)\right] e^{-\mu_{j} r_{-}^{2} / 2}\right. \\
&\left.+e^{-\mu_{i} r_{-}^{2} / 2}\left[\left(\boldsymbol{\sigma} \cdot \mathbf{r}_{-}\right)\left(\boldsymbol{\sigma} \cdot \hat{\mathbf{r}}_{ \pm}\right)\right] e^{-\mu_{j} r_{+}^{2} / 2}\right\}
\end{aligned}
$$

In addition to Eq. A36 we also need

$$
\left(\boldsymbol{\sigma} \cdot \mathbf{r}_{+}\right)\left(\boldsymbol{\sigma} \cdot \hat{\mathbf{r}}_{ \pm}\right)=r_{+}\left(\hat{\mathbf{r}}_{+} \cdot \hat{\mathbf{r}}_{ \pm}\right)
$$

which becomes $r_{+}$for the $z<0$ integration and $\left(\rho^{2}+z^{2}-\delta^{2}\right) / \sqrt{\rho^{2}+(z-\delta)^{2}}$ for the $z>0$ integration.

The integrations over $z$ go much easier if one re-defines the integrations over $z$ in terms of $\mu=\mu_{i}+\mu_{j}$ and $\nu=\mu_{i}-\mu_{j}$. The resulting integrals in $\mu$ and $\nu$ can then be converted back to $\mu_{i}$ and $\mu_{j}$. We find

$$
\begin{aligned}
& K_{i j,<\nabla V S>}^{(1)}= \frac{1}{\mu_{i} \mu_{j}\left(\mu_{i}+\mu_{j}\right)^{2}}\left[2 \mu_{j}\left(\mu_{j}-\mu_{i}\right) e^{-2 \mu_{i} \delta^{2}}+2 \mu_{i}\left(\mu_{i}-\mu_{j}\right) e^{-2 \mu_{j} \delta^{2}}\right. \\
&-\left.-\left(\mu_{i}-\mu_{j}\right)^{2} e^{-\left(\mu_{i}+\mu_{j}\right) \delta^{2} / 2}\right] \\
&-\frac{1}{2 \delta \mu_{i}^{2} \mu_{j}^{2}}\left[\frac{\pi}{2\left(\mu_{i}+\mu_{j}\right)^{5}}\right]^{1 / 2} \times \\
&\left\{\left(\mu_{i}+\mu_{j}\right)^{3}\left(\mu_{i}+\mu_{j}-2 \mu_{i} \mu_{j} \delta^{2}\right) \operatorname{Erfc}\left(\sqrt{\left(\mu_{i}+\mu_{j}\right) / 2} \delta\right)\right.
\end{aligned}
$$




$$
\begin{gathered}
+2 \mu_{i}^{2}\left[\left(\mu_{i}^{2}+4 \mu_{i} \mu_{j}+3 \mu_{j}^{2}\right)-4 \mu_{j}^{2}\left(\mu_{i}-\mu_{j}\right) \delta^{2}\right] \\
\times e^{-2 \mu_{i} \mu_{j} \delta^{2} /\left(\mu_{i}+\mu_{j}\right)} \operatorname{Erf}\left(\sqrt{2 /\left(\mu_{i}+\mu_{j}\right)} \mu_{j} \delta\right) \\
-2 \mu_{j}^{2}\left[3 \mu_{i}^{2}+4 \mu_{i} \mu_{j}+\mu_{j}^{2}-4 \mu_{i}^{2}\left(\mu_{j}-\mu_{i}\right) \delta^{2}\right] \\
\times e^{-2 \mu_{i} \mu_{j} \delta^{2} /\left(\mu_{i}+\mu_{j}\right)} \operatorname{Erfc}\left(\sqrt{2 /\left(\mu_{i}+\mu_{j}\right)} \mu_{i} \delta\right) \\
-\left[\left(\mu_{i}^{3}+5 \mu_{i}^{2} \mu_{j}+5 \mu_{i} \mu_{j}^{2}+\mu_{j}^{3}\right)-8 \mu_{i}^{2} \mu_{j}^{2} \delta^{2}\right] \\
\left.\times\left(\mu_{i}-\mu_{j}\right) e^{\left.-2 \mu_{i} \mu_{j}\right) \delta^{2} /\left(\mu_{i}+\mu_{j}\right)} \operatorname{Erfc}\left(\frac{\left(\mu_{i}-\mu_{j}\right) \delta}{\sqrt{2\left(\mu_{i}+\mu_{j}\right)}}\right)\right\},
\end{gathered}
$$

which also is symmetric and goes to $-8 /\left(\mu_{i}+\mu_{j}\right)^{2}=2 K_{i j,<\nabla V S>}^{(0)}$ at $\delta=0$. This integral does have some odd terms in $\delta$. As a function of $\delta$ it resembles a Gaussian, i.e., looks like that shown in Fig. 91 A.

Because $K_{i j,<\nabla V S>}^{(1)}=K_{j i,<\nabla V S>}^{(1)}$ we can again finally write

$$
<[\nabla V S]^{(1)}>=\sum_{i, j}\left(a_{j} b_{i}+a_{i} b_{j}\right) \quad K_{i j,<\nabla V S>}^{(1)},
$$

mirroring the form of Eq. A39).

\section{The off-diagonal expectation $-2 i V(\mathbf{r}) \boldsymbol{\alpha} \cdot \nabla$}

For this off-diagonal operator $\mathbf{X}_{12}=\mathbf{X}_{21}=-2 V(\mathbf{r}) \nabla$ in Eq. A8) and the direct term expectation, Eq. (A9), has all four terms

$$
\begin{array}{rl}
<[2 V \nabla]^{(0)}>=\frac{1}{8 \pi} \sum_{i, j} \int d^{3} r & V(\mathbf{r})\left\{e ^ { - \mu _ { i } r _ { - } ^ { 2 } / 2 } \left[2 a_{i} b_{j}(\boldsymbol{\sigma} \cdot \nabla)\left(\boldsymbol{\sigma} \cdot \mathbf{r}_{-}\right)\right.\right. \\
& \left.-2 b_{i} a_{j}\left(\boldsymbol{\sigma} \cdot \mathbf{r}_{-}\right)(\boldsymbol{\sigma} \cdot \nabla)\right] e^{-\mu_{j} r_{-}^{2} / 2} \\
+e^{-\mu_{j} r_{-}^{2} / 2}\left[2 a_{j} b_{i}(\boldsymbol{\sigma} \cdot \nabla)\left(\boldsymbol{\sigma} \cdot \mathbf{r}_{-}\right)\right. \\
- & \left.\left.2 b_{j} a_{i}\left(\boldsymbol{\sigma} \cdot \mathbf{r}_{-}\right)(\boldsymbol{\sigma} \cdot \nabla)\right] e^{-\mu_{i} r_{-}^{2} / 2}\right\} .
\end{array}
$$

With

$$
\nabla_{k} e^{-\mu_{i} r_{-}^{2} / 2}=-\mu_{i}\left(\mathbf{r}_{-}\right)_{k} e^{-\mu_{i} r_{-}^{2} / 2}, \quad \nabla_{k}\left(\mathbf{r}_{-}\right)_{l}=\delta_{k l}, \quad \text { and } \quad(\boldsymbol{\sigma} \cdot \nabla)\left(\boldsymbol{\sigma} \cdot \mathbf{r}_{-}\right)=3
$$

we have, for the first terms in the square brackets of Eq. A45,

$$
\begin{gathered}
(\boldsymbol{\sigma} \cdot \nabla)\left(\boldsymbol{\sigma} \cdot \mathbf{r}_{-}\right) e^{-\mu_{i} r_{-}^{2} / 2}=e^{-\mu_{i} r_{-}^{2} / 2}(\boldsymbol{\sigma} \cdot \nabla)\left(\boldsymbol{\sigma} \cdot \mathbf{r}_{-}\right)+\boldsymbol{\sigma} \cdot\left[\left(\boldsymbol{\sigma} \cdot \mathbf{r}_{-}\right) \nabla e^{-\mu_{i} r_{-}^{2} / 2}\right] \\
=\left[3-\mu_{i}\left(\boldsymbol{\sigma} \cdot \mathbf{r}_{-}\right)\left(\boldsymbol{\sigma} \cdot \mathbf{r}_{-}\right)\right] e^{-\mu_{i} r_{-}^{2} / 2}=\left(3-\mu_{i} r_{-}^{2}\right) e^{-\mu_{i} r_{-}^{2} / 2}
\end{gathered}
$$


and similarly when acting on $e^{-\mu_{j} r_{-}^{2} / 2}$.

For the second terms in the square brackets of Eq. A45,

$$
\left(\boldsymbol{\sigma} \cdot \mathbf{r}_{-}\right)(\boldsymbol{\sigma} \cdot \nabla) e^{-\mu_{i} r_{-}^{2} / 2}=-\mu_{i}\left(\boldsymbol{\sigma} \cdot \mathbf{r}_{-}\right)\left(\boldsymbol{\sigma} \cdot \mathbf{r}_{-}\right) e^{-\mu_{i} r_{-}^{2} / 2}=-\mu_{i} r_{-}^{2} e^{-\mu_{i} r_{-}^{2} / 2}
$$

and, again, similarly when acting on $e^{-\mu_{j} r_{-}^{2} / 2}$.

With Eqs. A47) and (A48), Eq. A45 reduces to

$$
\begin{aligned}
<[2 V \nabla]^{(0)}>= & \frac{1}{4 \pi} \sum_{i, j} \int d^{3} r\left\{e^{-\mu_{i} r_{-}^{2} / 2} V\left(r_{ \pm}\right)\left[a_{i} b_{j}\left(3-\mu_{j} r_{-}^{2}\right)+b_{i} a_{j} \mu_{j} r_{-}^{2}\right] e^{-\mu_{j} r_{-}^{2} / 2}\right. \\
& \left.+e^{-\mu_{j} r_{-}^{2} / 2} V\left(r_{ \pm}\right)\left[a_{j} b_{i}\left(3-\mu_{i} r_{-}^{2}\right)+b_{j} a_{i} \mu_{i} r_{-}^{2}\right] e^{-\mu_{j} r_{-}^{2} / 2}\right\} \\
= & \sum_{i, j}\left\{a_{i} b_{j} K_{i j,<2 V \nabla>}^{(0)}+a_{j} b_{i} K_{j i,<2 V \nabla>}^{(0)}\right\}
\end{aligned}
$$

where, with $V\left(r_{ \pm}\right)=r_{ \pm}-R$

$$
\begin{aligned}
K_{i j,<2 V \nabla>}^{(0)} & =\frac{1}{4 \pi} \int d^{3} r e^{-\mu_{i} r_{-}^{2} / 2}\left(r_{ \pm}-R\right)\left[\left(\mu_{i}-\mu_{j}\right) r_{-}^{2}-3\right] e^{-\mu_{j} r_{-}^{2} / 2} . \\
& =\left(\mu_{i}-\mu_{j}\right) K_{i j, a}^{(0)}-\left(\mu_{i}-\mu_{j}\right) R K_{i j, b}^{(0)}+3 K_{i j, c}^{(0)}-3 R K_{i j, d}^{(0)}
\end{aligned}
$$

where these four integrals are

$$
\begin{aligned}
K_{i j, a}^{(0)}= & \frac{1}{4 \pi} \int d^{3} r e^{-\mu_{i} r_{-}^{2} / 2} r_{ \pm} r_{-}^{2} e^{-\mu_{j} r_{-}^{2} / 2} \\
= & \frac{1}{2\left(\mu_{j}+\mu_{i}\right)^{3}}\left[16+6 e^{-2\left(\mu_{j}+\mu_{i}\right) \delta^{2}}-11 e^{-\left(\mu_{j}+\mu_{i}\right) \delta^{2} / 2}\right] \\
& +\frac{1}{2 \delta}\left[\frac{\pi}{2\left(\mu_{j}+\mu_{i}\right)^{7}}\right]^{1 / 2}\left\{\left[5+9\left(\mu_{j}+\mu_{i}\right) \delta^{2}\right] \operatorname{Erfc}\left(\sqrt{\left(\mu_{j}+\mu_{i}\right) / 2} \delta\right)\right. \\
& \left.-\left[5+12\left(\mu_{j}+\mu_{i}\right) \delta^{2}\right] \operatorname{Erfc}\left(\sqrt{2\left(\mu_{j}+\mu_{i}\right)} \delta\right)\right\}, \\
K_{i j, b}^{(0)}= & \frac{1}{4 \pi} \int d^{3} r e^{-\mu_{i} r_{-}^{2} / 2} r_{-}^{2} e^{-\mu_{j} r_{-}^{2} / 2}=3\left[\frac{\pi}{2\left(\mu_{j}+\mu_{i}\right)^{5}}\right]^{1 / 2}, \\
K_{i j, c}^{(0)}= & \frac{1}{4 \pi} \int d^{3} r e^{-\mu_{i} r_{-}^{2} / 2} r_{ \pm} e^{-\mu_{j} r_{-}^{2} / 2}=I_{i j,<r_{ \pm}>}^{(0)}, \\
K_{i j, d}^{(0)}= & \frac{1}{4 \pi} \int d^{3} r e^{-\mu_{i} r_{-}^{2} / 2} e^{-\mu_{j} r_{-}^{2} / 2}=I_{i j,<1>}^{(0)} \cdot
\end{aligned}
$$

$K_{i j, a}^{(0)}$ has an odd term in $\delta$ and its plot resembles that shown in Fig. 9D. All four of the above integrals are symmetric in $i$ and $j$, so we can finally write

$$
<[2 V \nabla]^{(0)}>=\sum_{i, j}\left(a_{j} b_{i}+a_{i} b_{j}\right) \quad K_{i j,<2 V \nabla>}^{(0)}=2 \sum_{i, j} a_{j} b_{i} K_{i j,<2 V \nabla>}^{(0)}
$$


For the cross term, from Eqs. A47) and (A48) and the like, Eq. A10 becomes

$$
\begin{gathered}
<[2 V \nabla]^{(1)}>=\frac{1}{8 \pi} \sum_{i, j} \int d^{3} r V\left(r_{ \pm}\right) \times \\
\left\{e^{-\mu_{i} r_{+}^{2} / 2}\left[2 a_{i} b_{j}(\boldsymbol{\sigma} \cdot \nabla)\left(\boldsymbol{\sigma} \cdot \mathbf{r}_{-}\right)-2 b_{i} a_{j}\left(\boldsymbol{\sigma} \cdot \mathbf{r}_{+}\right)(\boldsymbol{\sigma} \cdot \nabla)\right] e^{-\mu_{j} r_{-}^{2} / 2}\right. \\
+e^{-\mu_{i} r_{-}^{2} / 2}\left[2 a_{i} b_{j}(\boldsymbol{\sigma} \cdot \nabla)\left(\boldsymbol{\sigma} \cdot \mathbf{r}_{+}\right)-2 b_{i} a_{j}\left(\boldsymbol{\sigma} \cdot \mathbf{r}_{-}\right)(\boldsymbol{\sigma} \cdot \nabla)\right] e^{-\mu_{j} r_{+}^{2} / 2} \\
+e^{-\mu_{j} r_{+}^{2} / 2}\left[2 a_{j} b_{i}(\boldsymbol{\sigma} \cdot \nabla)\left(\boldsymbol{\sigma} \cdot \mathbf{r}_{-}\right)-2 b_{j} a_{i}\left(\boldsymbol{\sigma} \cdot \mathbf{r}_{+}\right)(\boldsymbol{\sigma} \cdot \nabla)\right] e^{-\mu_{i} r_{-}^{2} / 2} \\
\left.+e^{-\mu_{j} r_{-}^{2} / 2}\left[2 a_{j} b_{i}(\boldsymbol{\sigma} \cdot \nabla)\left(\boldsymbol{\sigma} \cdot \mathbf{r}_{+}\right)-2 b_{j} a_{i}\left(\boldsymbol{\sigma} \cdot \mathbf{r}_{-}\right)(\boldsymbol{\sigma} \cdot \nabla)\right] e^{-\mu_{i} r_{+}^{2} / 2}\right\} \\
=\frac{1}{4 \pi} \sum_{i, j} \int d^{3} r V\left(r_{ \pm}\right)\left\{e^{-\mu_{i} r_{+}^{2} / 2}\left[a_{i} b_{j}\left(3-\mu_{j} r_{-}^{2}\right)+b_{i} a_{j} \mu_{j}\left(\mathbf{r}_{+} \cdot \mathbf{r}_{-}\right)\right] e^{-\mu_{j} r_{-}^{2} / 2}\right. \\
\quad+e^{-\mu_{i} r_{-}^{2} / 2}\left[a_{i} b_{j}\left(3-\mu_{j} r_{+}^{2}\right)+b_{i} a_{j} \mu_{j}\left(\mathbf{r}_{+} \cdot \mathbf{r}_{-}\right)\right] e^{-\mu_{j} r_{+}^{2} / 2} \\
\quad+e^{-\mu_{j} r_{+}^{2} / 2}\left[a_{j} b_{i}\left(3-\mu_{i} r_{-}^{2}\right)+b_{j} a_{i} \mu_{i}\left(\mathbf{r}_{+} \cdot \mathbf{r}_{-}\right)\right] e^{-\mu_{i} r_{-}^{2} / 2} \\
\left.\quad+e^{-\mu_{j} r_{-}^{2} / 2}\left[a_{j} b_{i}\left(3-\mu_{i} r_{+}^{2}\right)+b_{j} a_{i} \mu_{i}\left(\mathbf{r}_{+} \cdot \mathbf{r}_{-}\right)\right] e^{-\mu_{i} r_{+}^{2} / 2}\right\} \\
=\sum_{i, j}\left\{a_{i} b_{j} K_{i j,<2 V \nabla>}^{(1)}+a_{j} b_{i} K_{j i,<2 V \nabla>}^{(1)}\right\}
\end{gathered}
$$

where

$$
\begin{aligned}
K_{i j,<2 V \nabla>}^{(1)}= & \frac{1}{4 \pi} \int d^{3} r\left(r_{ \pm}-R\right)\left\{e^{-\mu_{i} r_{+}^{2} / 2}\left[\left(3-\mu_{j} r_{-}^{2}\right)+\mu_{i}\left(\mathbf{r}_{+} \cdot \mathbf{r}_{-}\right)\right] e^{-\mu_{j} r_{-}^{2} / 2}\right. \\
& \left.+e^{-\mu_{i} r_{-}^{2} / 2}\left[\left(3-\mu_{j} r_{+}^{2}\right)+\mu_{i}\left(\mathbf{r}_{+} \cdot \mathbf{r}_{-}\right)\right] e^{-\mu_{j} r_{+}^{2} / 2}\right\} \\
= & -\mu_{j} K_{i j, a}^{(1)}+\mu_{j} R K_{i j, b}^{(1)}+\mu_{i} K_{i j, c}^{(1)}-\mu_{i} R K_{i j, d}^{(1)}+3 K_{i j, e}^{(1)}-3 R K_{i j, f}^{(1)} .
\end{aligned}
$$

The first integral,

$$
K_{i j, a}^{(1)}=\frac{1}{4 \pi} \int d^{3} r\left\{e^{-\mu_{i} r_{+}^{2} / 2} r_{ \pm} r_{-}^{2} e^{-\mu_{j} r_{-}^{2} / 2}+e^{-\mu_{i} r_{-}^{2} / 2} r_{ \pm} r_{+}^{2} e^{-\mu_{j} r_{+}^{2} / 2}\right\}
$$

can be done using $\mu=\mu_{i}+\mu_{j}$ and $\nu=\mu_{i}-\mu_{j}$, noting that $\mu>|\nu|$. Writing

$$
\begin{aligned}
e^{-\mu_{i} r_{+}^{2} / 2} r_{-}^{2} & e^{-\mu_{j} r_{-}^{2} / 2}+e^{-\mu_{i} r_{-}^{2} / 2} r_{+}^{2} e^{-\mu_{j} r_{+}^{2} / 2} \\
& =2 e^{-\mu\left(\rho^{2}+z^{2}+\delta^{2}\right) / 2}\left\{\left(\rho^{2}+z^{2}+\delta^{2}\right) \cosh (\nu \delta z)+(2 z \delta) \sinh (\nu \delta z)\right\}
\end{aligned}
$$

displays the $i, j$ symmetric and anti-symmetric parts explicitly. After converting back to $\mu_{i}$ and $\mu_{j}$,

$$
\begin{aligned}
K_{i j, a}^{(1)}=\frac{2}{\mu_{j}\left(\mu_{i}+\mu_{j}\right)^{4}} & \left\{\left[5 \mu_{j}\left(\mu_{i}+\mu_{j}\right)+4 \mu_{i}^{2} \mu_{j} \delta^{2}\right] e^{-2 \mu_{i} \delta^{2}}\right. \\
+ & {\left[\left(\mu_{i}+\mu_{j}\right)\left(-2 \mu_{i}+3 \mu_{j}\right)+4 \mu_{i}^{2} \mu_{j} \delta^{2}\right] e^{-2 \mu_{j} \delta^{2}} } \\
+ & {\left.\left[\left(\mu_{i}+\mu_{j}\right)\left(\mu_{i}-4 \mu_{j}\right)-4 \mu_{i}^{2} \mu_{j} \delta^{2}\right] e^{-\frac{1}{2}\left(\mu_{i}+\mu_{j}\right) \delta^{2}}\right\} }
\end{aligned}
$$




$$
\begin{aligned}
& +\frac{1}{2 \delta \mu_{i} \mu_{j}^{2}}\left[\frac{\pi}{2\left(\mu_{i}+\mu_{j}\right)^{9}}\right]^{1 / 2} \times \\
& \begin{array}{c}
2\left[\mu_{i}\left(\mu_{i}+\mu_{j}\right)^{2}\left(2 \mu_{i}+5 \mu_{j}\right)+4 \mu_{i} \mu_{j}\left(\mu_{i}+\mu_{j}\right)\left(\mu_{i}^{2}-2 \mu_{i} \mu_{j}+3 \mu_{j}^{2}\right) \delta^{2}+16 \mu_{i}^{3} \mu_{j}^{3} \delta^{4}\right] \\
\quad \times e^{-2 \mu_{i} \mu_{j} \delta^{2} /\left(\mu_{i}+\mu_{j}\right)} \operatorname{Erf}\left(\sqrt{2 /\left(\mu_{i}+\mu_{j}\right)} \mu_{j} \delta\right)
\end{array} \\
& -2 \mu_{j}^{2}\left[3\left(\mu_{i}+\mu_{j}\right)^{2}+24 \mu_{i}^{2}\left(\mu_{i}+\mu_{j}\right) \delta^{2}+16 \mu_{i}^{4} \delta^{4}\right] \\
& \quad \times e^{-2 \mu_{i} \mu_{j} \delta^{2} /\left(\mu_{i}+\mu_{j}\right)} \operatorname{Erfc}\left(\sqrt{2 /\left(\mu_{i}+\mu_{j}\right)} \mu_{i} \delta\right) \\
& -\left[\left(\mu_{i}+\mu_{j}\right)^{2}\left(2 \mu_{i}^{2}+5 \mu_{i} \mu_{j}-3 \mu_{j}^{2}\right)+4 \mu_{i} \mu_{j}\left(\mu_{i}+\mu_{j}\right)\left(\mu_{i}^{2}-8 \mu_{i} \mu_{j}+3 \mu_{j}^{2}\right) \delta^{2}\right. \\
& \left.\quad-16 \mu_{i}^{3} \mu_{j}^{2}\left(\mu_{i}-\mu_{j}\right) \delta^{4}\right] e^{-2 \mu_{i} \mu_{j} \delta^{2} /\left(\mu_{i}+\mu_{j}\right)} \operatorname{Erfc}\left(\frac{\left(\mu_{i}-\mu_{j}\right)}{\sqrt{2\left(\mu_{i}+\mu_{j}\right)}} \delta\right) \\
& \left.+\left(\mu_{i}+\mu_{j}\right)^{3}\left(2 \mu_{i}+3 \mu_{j}\right) \operatorname{Erfc}\left(\sqrt{\left(\mu_{i}+\mu_{j}\right) / 2} \delta\right)\right\}
\end{aligned}
$$

which is, as expected, not symmetric in $i$ and $j$. It is, however, non-singular: $K_{i j, a}^{(1)}=$ $16 /\left(\mu_{i}+\mu_{j}\right)^{3}$ at $\delta=0$. Its plot resembles that in Fig. 9D.

The second integral is much simpler,

$$
\begin{aligned}
K_{i j, b}^{(1)} & =\frac{1}{4 \pi} \int d^{3} r\left\{e^{-\mu_{i} r_{+}^{2} / 2} r_{-}^{2} e^{-\mu_{j} r_{-}^{2} / 2}+e^{-\mu_{i} r_{-}^{2} / 2} r_{+}^{2} e^{-\mu_{j} r_{+}^{2} / 2}\right\} \\
& =\left[\frac{2 \pi}{\left(\mu_{i}+\mu_{j}\right)^{7}}\right]^{1 / 2}\left[3\left(\mu_{i}+\mu_{j}\right)+4 \mu_{j}^{2} \delta^{2}\right] e^{-2 \mu_{i} \mu_{j} \delta^{2} /\left(\mu_{i}+\mu_{j}\right)},
\end{aligned}
$$

which is also non-symmetric, but only because of the term proportional to $\delta^{2}$. As a function of $\delta$ it looks like Fig. $9 \mathrm{E}$. 
Almost as complicated as $K_{i j, a}^{(1)}$, the third integral is

$$
\begin{aligned}
& K_{i j, c}^{(1)}=\frac{1}{4 \pi} \int d^{3} r\left\{e^{-\mu_{j} r_{+}^{2} / 2} r_{ \pm}\left(\mathbf{r}_{+} \cdot \mathbf{r}_{-}\right) e^{-\mu_{i} r_{-}^{2} / 2}\right. \\
& \left.+e^{-\mu_{j} r_{-}^{2} / 2} r_{ \pm}\left(\mathbf{r}_{+} \cdot \mathbf{r}_{-}\right) e^{-\mu_{i} r_{+}^{2} / 2}\right\} \\
& =\frac{1}{\mu_{i} \mu_{j}\left(\mu_{i}+\mu_{j}\right)^{4}}\left\{2 \mu_{j}\left[\left(\mu_{i}+\mu_{j}\right)\left(4 \mu_{i}-\mu_{j}\right)-4 \mu_{i}^{2} \mu_{j} \delta^{2}\right] e^{-2 \mu_{i} \delta^{2}}\right. \\
& +2 \mu_{i}\left[\left(\mu_{i}+\mu_{j}\right)\left(4 \mu_{j}-\mu_{i}\right)-4 \mu_{i} \mu_{j}^{2} \delta^{2}\right] e^{-2 \mu_{j} \delta^{2}} \\
& \left.+\left[\left(\mu_{i}+\mu_{j}\right)\left(\mu_{i}^{2}-8 \mu_{i} \mu_{j}+\mu_{j}^{2}\right)+8 \mu_{i}^{2} \mu_{j}^{2} \delta^{2}\right] e^{-\frac{1}{2}\left(\mu_{i}+\mu_{j}\right) \delta^{2}}\right\} \\
& +\frac{1}{2 \delta \mu_{i}^{2} \mu_{j}^{2}}\left[\frac{\pi}{2\left(\mu_{i}+\mu_{j}\right)^{9}}\right]^{1 / 2} \times \\
& \left\{2 \mu_{j}^{2}\left[\left(\mu_{i}+\mu_{j}\right)^{2}\left(4 \mu_{i}+\mu_{j}\right)+8 \mu_{i}^{2}\left(\mu_{i}+\mu_{j}\right)\left(2 \mu_{i}-\mu_{j}\right) \delta^{2}-16 \mu_{i}^{4} \mu_{j} \delta^{4}\right]\right. \\
& \times e^{-2 \mu_{i} \mu_{j} \delta^{2} /\left(\mu_{i}+\mu_{j}\right)} \operatorname{Erf}\left(\sqrt{\frac{2}{\mu_{i}+\mu_{j}}} \mu_{i} \delta\right) \\
& -2 \mu_{i}^{2}\left[\left(\mu_{i}+\mu_{j}\right)^{2}\left(4 \mu_{j}+\mu_{i}\right)+8 \mu_{j}^{2}\left(\mu_{i}+\mu_{j}\right)\left(2 \mu_{j}-\mu_{i}\right) \delta^{2}-16 \mu_{i} \mu_{j}^{4} \delta^{4}\right] \\
& \times e^{-2 \mu_{i} \mu_{j} \delta^{2} /\left(\mu_{i}+\mu_{j}\right)} \operatorname{Erfc}\left(\sqrt{\frac{2}{\mu_{i}+\mu_{j}}} \mu_{j} \delta\right) \\
& +\left[\left(\mu_{i}+\mu_{j}\right)^{2}\left(\mu_{i}^{2}+5 \mu_{i} \mu_{j}+\mu_{j}^{2}\right)-24 \mu_{i}^{2} \mu_{j}^{2}\left(\mu_{i}+\mu_{j}\right) \delta^{2}+16 \mu_{i}^{3} \mu_{j}^{3} \delta^{4}\right] \\
& \times\left(\mu_{i}-\mu_{j}\right) e^{-2 \mu_{i} \mu_{j} \delta^{2} /\left(\mu_{i}+\mu_{j}\right)}\left[1+\operatorname{Erf}\left(\frac{\left(\mu_{i}-\mu_{j}\right)}{\sqrt{2\left(\mu_{i}+\mu_{j}\right)}} \delta\right)\right] \\
& +\left(\mu_{i}+\mu_{j}\right)^{3}\left[\left(\mu_{i}^{2}+3 \mu_{i} \mu_{j}+\mu_{j}^{2}\right)-2 \mu_{i} \mu_{j}\left(\mu_{i}+\mu_{j}\right) \delta^{2}\right] \\
& \left.\times \operatorname{Erfc}\left(\sqrt{\left(\mu_{i}+\mu_{j}\right) / 2} \delta\right)\right\},
\end{aligned}
$$

which is surprisingly both symmetric, $K_{j i, c}^{(1)}=K_{i j, c}^{(1)}$, and non-singular: $K_{i j, c}^{(1)}=16 /\left(\mu_{i}+\mu_{j}\right)^{3}$ at $\delta=0$. This integral as a function of $\delta$ looks like Fig. $9 \mathrm{~B}$.

The fourth integral is also simple,

$$
\begin{aligned}
K_{i j, d}^{(1)} & =\frac{1}{4 \pi} \int d^{3} r\left\{e^{-\mu_{j} r_{+}^{2} / 2}\left(\mathbf{r}_{+} \cdot \mathbf{r}_{-}\right) e^{-\mu_{i} r_{-}^{2} / 2}+e^{-\mu_{j} r_{-}^{2} / 2}\left(\mathbf{r}_{+} \cdot \mathbf{r}_{-}\right) e^{-\mu_{i} r_{+}^{2} / 2}\right\} \\
& =\left[\frac{2 \pi}{\left(\mu_{j}+\mu_{i}\right)^{7}}\right]^{1 / 2}\left[3\left(\mu_{j}+\mu_{i}\right)-4 \mu_{i} \mu_{j} \delta^{2}\right] e^{-2 \mu_{i} \mu_{j} \delta^{2} /\left(\mu_{i}+\mu_{j}\right)} .
\end{aligned}
$$

Its $\delta$ dependence, Fig. $9 \mathrm{~F}$, shows a relatively deeper minimum than that depicted in Fig. 9B. The fifth and sixth integrals are already familiar,

$$
\begin{aligned}
& K_{i j, e}^{(1)}=\frac{1}{4 \pi} \int d^{3} r\left\{e^{-\mu_{i} r_{+}^{2} / 2} r_{ \pm} e^{-\mu_{j} r_{-}^{2} / 2}+e^{-\mu_{i} r_{-}^{2} / 2} r_{ \pm} e^{-\mu_{j} r_{+}^{2} / 2}\right\}=I_{i j,<r_{ \pm}>}^{(1)} \\
& K_{i j, f}^{(1)}=\frac{1}{4 \pi} \int d^{3} r\left\{e^{-\mu_{i} r_{+}^{2} / 2} e^{-\mu_{j} r_{-}^{2} / 2}+e^{-\mu_{i} r_{-}^{2} / 2} e^{-\mu_{j} r_{+}^{2} / 2}\right\}=I_{i j,<1>}^{(1)} .
\end{aligned}
$$


These last three integrals, $K_{i j, d}^{(1)}$ through $K_{i j, f}^{(1)}$, are all symmetric in $i$ and $j$. 